\title{
High-Frequency Tail Index Estimation by Nearly Tight Frames
}

\author{
Claudio Durastanti and Xiaohong Lan
}

\begin{abstract}
This work develops the asymptotic properties (weak consistency and Gaussianity), in the high-frequency limit, of approximate maximum likelihood estimators for the spectral parameters of Gaussian and isotropic spherical random fields. The procedure we used exploits the so-called mexican needlet construction by Geller and Mayeli in [21. Furthermore, we propose a plug-in procedure to optimize the precision of the estimators in terms of asymptotic variance.
\end{abstract}

\section{Introduction}

The aim of this paper is to investigate the asymptotic behaviour of a Whittlelike approximate maximum likelihood estimates of the spectral parameters (e.g., the spectral index) of isotropic Gaussian random fields defined on the unit sphere $\mathbb{S}^{2}$. We employ a procedure based on the so-called mexican needlet construction by Geller and Mayeli in [21]. Furthermore, we develop a plug-in procedure aimed to merge and to optimize these results with the achievements pursued in $\mathbf{1 2}$, 13, see also [14, where the asymptotic behaviour of Whittle-like estimates were studied respectively in the harmonic and standard needlet analysis frameworks.

Under the hypothesis of Gaussianity, fixing smoothness conditions on the behaviour of the angular power spectrum, we pursue weak consistency and central limit theorem allowing for feasible inference. From the technical point of view, the asymptotic framework we use here is rather different from the usual, being based on observations collected at higher and higher frequencies on a fixed-domain (i. e. the unit sphere). In this sense, this work can be related to the area of fixed-domain asymptotics (see for instance [2], 34]); on the other hand, as for [12] and [13], some of the techniques used here are close to those adopted by [46] to analyze the asymptotic behaviour of the semiparametric estimates of the long memory parameter for the covariance of stationary processes. In terms of the angular power spectrum, we shall also focus on semiparametric models where only the high-frequency/smallscale behaviour of the random field is constrained. In particular, we consider both

2010 Mathematics Subject Classification. Primary 62M15, 62M30; Secondary 60G60, 42C40.

Key words and phrases. Spherical Random Fields, high frequency asymptotics, Whittle likelihood, mexican needlets, parametric and semiparametric estimates .

Research partially supported by ERC Grant n. 277742 Pascal. 
full-band and narrow-band estimates, where the latter allow unbiased estimation under more general assumption, by paying the price of a slower rate of convergence if compared to the former.

This investigation, as many others regarding statistical inference on spherical random fields, is strongly motivated by practical applications, especially in cosmology and astrophysics (see for instance [37 and the references therein). For instance, as described in $\mathbf{9}$ and $[\mathbf{8}$, satellite missions such as WMAP and Planck are now providing huge datasets on Cosmic Microwave Background (CMB) radiation, usually assumed to be a realization of an isotropic, Gaussian spherical random field: the issues concerning parameter estimation have been considered by many applied papers (see 24, 31 for a review), but in our knowledge, until now, rigorous asymptotic results are still missing in literature. We however refer also to [4, 15, [19, 43, 44, 36 for further theoretical and applied results on angular power spectrum estimation in nonparametric settings, and to [25, [27, [26, [28, [32, 29 and 37 for further results on statistical inference for spherical random fields or wavelets applied to CMB radiation.

Another result we work out in this paper concerns the formulation of a plug-in procedure which combines the application of the asymptotic results here attained with those described in $\mathbf{1 2}$ and $\mathbf{1 3}$, where the authors proved that weak consistency and central limit theorem can be achieved respectively by standard Fourier and standard spherical needlet analysis. In [12, the authors themselves have put in evidence that, if the asymptotic achievements are better with respect to those obtain in needlet framework in terms of precision of the estimates (e.g. their asymptotic variance is smaller), in many practical circumstances the implementation of spherical harmonics estimates may present some difficulties, due to their lack of localization in real space. The presence of unobserved regions on the sphere (common situation in the case of Cosmological applications), can indeed make their implementation infeasible, and spherical harmonics exclude the possibility of separate estimation on different hemispheres, as considered for instance by [5, 45. In view of these issues, in [13, the authors investigated the Whittle-like procedures to a spherical wavelet framework, in order to exploit the double-localization properties (in real and harmonic space) of such constructions, at the cost of a smaller precision in term of convergence in law of the estimates. They focussed their attention on spherical needlets, second-generation wavelets on the sphere, introduced in 2006 by [40] and [41, and very extensively exploited both in the statistical literature and for astrophysical applications in the last few years: for instance, their stochastic properties are developed in [4, [5], 6 [29, 30] and [39. More recently, needlets have been generalized in different ways: we cite spin needlets (see [17]), and mixed needlets (cfr. [18), which represent the natural generalization to the case of spin fiber bundles, again developed in view of Cosmological applications such as weak gravitational lensing and the polarization of the Cosmic Microwave Background (CMB) radiation (see for instance [4, 8], 11, 15, [19, 16, 38, 44, 45, 47]). On the other hand, needlets have been generalized to an unbounded support in the frequency domain by [20, 21] and [22], the so-called Mexican needlets. In this case, as we will describe in details below, even if the support in frequency domain is unbounded, the form of the weight function, depending on the scale parameter $p$, is such that for each wavelet there is a small numbers of frequencies which give a contribution substantially far from zero, while in the real domain the 
same weight function allows a closer localization than the one related to standard spherical needlets. In particular this double localization depends on the value of $p$ or, better, on its distance from the spectral index, allowing these estimates to be more efficient than the ones obtained with standard needlets. Our idea, therefore, is to build a plug-in procedure on two steps, the first step being to estimate approximately the value of the spectral index by standard needlets and the second step providing a estimation with mexican needlets, whereas the value of the scale parameter $p$ will allow a more efficent estimator.

The plan of the paper is as follows: in Section 2, we will recall some background material on mexican needlet analysis for spherical isotropic random fields; in Section 3 we will introduce and describe the Whittle-like minimum contrast estimators, while in Section 4 we shall establish the asymptotic results on these estimators. In Section 5 we present results on narrow band estimates, while in Section 6 we will describe the plug-in procedure mentioned above. Finally, the appendix collects some analytical and statistical auxiliary results.

\section{Random fields and mexican needlets}

In this Section we will introduce the mexican needlet framework (for more details, cfr. 21]) and its application to the study of the isotropic, Gaussian random fields on the sphere. First of all, consider the set of spherical harmonics $\left\{Y_{l m}: l \geq 0, m=-l, \ldots, l\right\}$. As well-known, it represents an orthonormal basis for the class of square-integrable functions on the unit sphere space $L^{2}\left(\mathbb{S}^{2}\right)$ : the spherical harmonics are defined as the eigenfunctions of the spherical Laplacian $\Delta_{S^{2}}$ corresponding to eigenvalues $-l(l+1)$ (see, for more details and analytic expressions, 1 [49, 50, 37] and, for extensions, 33, 35). The mexican needlets are defined in 21] as

$$
\psi_{j k ; p}(x):=\sqrt{\lambda_{j k}} \sum_{l \geq 1} f_{p}\left(\frac{l}{B^{j}}\right) \sum_{m=-l}^{l} \bar{Y}_{l m}(x) Y_{l m}\left(\xi_{j k}\right)
$$

where

$$
f_{p}(x)=x^{2 p} \exp \left(-x^{2}\right)
$$

Observe that $\left\{\xi_{j k}\right\}$ is a set of cubature points on the sphere, indexed by resolution level index $j$ and the cardinality of the point over the fixed resolution level $k$, while $\lambda_{j k}>0$ corresponds to the weight associated to any $\xi_{j k}$. The scalar $N_{j}$ denotes the number of cubature points for a given level $j$ (cfr. [40], 41, see also e.g. [21] and 37 ), chosen to satisfy the following

$$
\lambda_{j k} \approx B^{-2 j}, N_{j} \approx B^{2 j},
$$

where by $a \approx b$, we mean that there exists $c_{1}, c_{2}>0$ such that $c_{1} a \leq b \leq c_{2} a$. Below, we shall assume for notational simplicity, as in [13, that there exists a positive constant $c_{B}$ such that $N_{j}=c_{B} B^{2 j}$ for all resolution levels $j$. In practice, cubature points and weights can be identified with those evaluated by common packages such as HealPix (see for instance [4, [10, [23]).

Considering $L_{l}(\langle x, y\rangle)=\sum_{m=-l}^{l} \bar{Y}_{l m}(x) Y_{l m}(y)$ as a projection operator, the definition (2.1) corresponds to a weighted convolution with a weight function (2.2): mexican needlets can be considered as an extension of the spherical standard needlets, proposed in [40, [41, see also [6], 12, $[\mathbf{3 7}$. The main difference between 
these two kinds of wavelets concerns their dependence on frequencies. In fact while standard needlets have a compact frequency support (see again [40, 41]), each mexican needlet is defined on the whole frequency range. In [21, mexican needlets are proved to form a nearly tight frame, differently from the standard needlets which describe a tight frame and, as consequence, are characterized by an exact reconstruction formula (see again [40]).

Consider now a zero-mean, isotropic Gaussian random fields $T: \mathbb{S}^{2} \times \Omega \rightarrow \mathbb{R}$; it is a well known fact that for every $g \in S O(3)$ and $x \in \mathbb{S}^{2}$, a field $T(\cdot)$ is isotropic if and only if

$$
T(x) \stackrel{d}{=} T(g x),
$$

where the equality holds in the sense of processes (see [36, [37), and that (see e.g. [37]) the following spectral representation holds:

$$
T(x)=\sum_{l \geq 0} \sum_{m=-l}^{l} a_{l m} Y_{l m}(x), a_{l m}=\int_{\mathbb{S}^{2}} T(x) \bar{Y}_{l m}(x) d x .
$$

Note that this equality holds in both $L^{2}\left(\mathbb{S}^{2} \times \Omega, d x \otimes \mathbb{P}\right)$ and $L^{2}(\mathbb{P})$ senses for every fixed $x \in \mathbb{S}^{2}$. For an isotropic Gaussian field, the spherical harmonics coefficients $a_{l m}$ are Gaussian complex random variables such that

$$
\mathbb{E}\left(a_{l m}\right)=0, \mathbb{E}\left(a_{l m} \bar{a}_{l_{1} m_{1}}\right)=\delta_{l}^{l_{1}} \delta_{m}^{m_{1}} C_{l} .
$$

The angular power spectrum $\left\{C_{l}, l=1,2,3, \ldots\right\}$ fully characterizes the dependence structure under Gaussianity. Properties of the spherical harmonics coefficients under Gaussianity and isotropy are discussed for instance by [3], [37; here we recall that

$$
\sum_{m=-l}^{l}\left|a_{l m}\right|^{2} \sim C_{l} \times \chi_{2 l+1}^{2} .
$$

Hence, given a realization of the random field, an estimator of the angular power spectrum can be defined as:

$$
\widehat{C}_{l}=\frac{1}{2 l+1} \sum_{m=-l}^{l}\left|a_{l m}\right|^{2},
$$

the empirical angular power spectrum. It is immediately observed that

$$
\mathbb{E}\left(\widehat{C}_{l}\right)=\frac{1}{2 l+1} \sum_{m=-l}^{l} C_{l}=C_{l}, \operatorname{Var}\left(\frac{\widehat{C}_{l}}{C_{l}}\right)=\frac{2}{2 l+1} \rightarrow 0 \text { for } l \rightarrow+\infty .
$$

As in [13, we introduce the following regularity condition on the angular power spectrum:

Condition 1 (Regularity). The random field $T(x)$ is Gaussian and isotropic with angular power spectrum $C_{l}$ so that for all $B>1$, there exist $\alpha_{0}>2, c_{0}>0$ such that:

$$
C_{l}=l^{-\alpha_{0}} G(l)>0, \text { for all } l \in \mathbb{N},
$$

where $c_{0}^{-1} \leq G(l) \leq c_{0}$ for all $l \in N$, and for every $r \in \mathbb{N}$, there exists $c_{r}>0$ such that:

$$
\left|\frac{d^{r}}{d u^{r}} G(u)\right| \leq c_{r} u^{-r}, \in(0,+\infty) .
$$


This assumption is fulfilled by popular physical models, for instance in a CMB framework the Sachs-Wolfe power spectrum, which is the leading model for fluctuations of the primordial gravitational potential, takes the form (2.5), see for instance 9 .

First of all, we stress that Condition 1 implies the following Condition 2 , given in 30 .

Condition 2. Condition 1 holds and, moreover, there exist $\alpha_{0}>2$ and a sequence of functions $\left\{g_{j}(\cdot)\right\}_{j=1,2, \ldots}$ such that:

$$
C_{l}=l^{-\alpha_{0}} g_{j}\left(\frac{l}{B^{j}}\right)>0, \text { for all } B^{j-1}<l<B^{j+1}, j=1,2 \ldots
$$

where $c_{0}^{-1} \leq g_{j} \leq c_{0}$ for all $j \in N$, and for every $r=0, \ldots, Q, Q \in \mathbb{N}$, there exists $c_{r}>0$ such that:

$$
\sup _{j} \sup _{B^{j-1}<u<B^{j+1}}\left|\frac{d^{r}}{d u^{r}} g_{j}(u)\right| \leq c_{r} .
$$

As an example, consider

$$
C_{l}=l^{-\alpha} \frac{P(l)}{Q(l)}
$$

where $P(l)=\sum_{i=1}^{p} c_{p, i} i^{i}$ and $Q(l)=\sum_{i=1}^{q} c_{q, i} l^{i}$ are positive polynomials of degree $p$ and $q$ respectively, so that $\alpha_{0}=\alpha-p+q>2$., so that

$$
\begin{aligned}
C_{l} & =l^{-\alpha+p-q} \frac{c_{p, p}}{c_{q, q}} \frac{1+\frac{c_{p, p-1}}{c_{p, p}} \frac{1}{l}+\frac{c_{p, p-2}}{c_{p, p}} \frac{1}{l^{2}}+\ldots}{\frac{c_{q, q-1}}{c_{q, q}} \frac{1}{l}+\frac{c_{q, q-}}{c_{q, q}} \frac{1}{l^{2}}+\ldots} \\
& =l^{-\alpha+p-q} \frac{c_{p, p}}{c_{q, q}} \frac{1+\frac{1}{B j} \frac{c_{p, p-1}}{c_{p, p}} \frac{B^{j}}{l}+\frac{1}{B^{2 j}} \frac{c_{p, p-2}}{c_{p, p}}\left(\frac{B^{j}}{l}\right)^{2}+\ldots \ldots}{B j} \frac{c_{q, q-1}}{c_{q, q}} \frac{1}{l} \frac{B^{j}}{l}+\frac{1}{B^{2 j}} \frac{c_{q, q-2}}{c_{q, q}} \frac{1}{l^{2}}\left(\frac{B^{j}}{l}\right)^{2}+\ldots \\
& =l^{-\alpha_{0}} g_{j}\left(\frac{l}{B^{j}}\right) .
\end{aligned}
$$

Condition 1 will be necessary to prove needlet coefficients (2.7) to be asymptotically uncorrelated (see [30], [39]); as we shall show, Condition 1 is sufficient to establish consistency for estimator we are going to define but we will consider two further nested assumptions, 3 (which implies and is implied by 1), to obtain asymptotic Gaussianity, and 4 (which implies 3) to provide a centered limiting distribution, see also [12, 13].

Condition 3. Condition 1 holds and moreover

$$
G(l)=G_{0}\left(1+\kappa l^{-1}+O\left(l^{-2}\right)\right) .
$$

Condition 4. Condition 1 holds and moreover

$$
G(l)=G_{0}\left(1+o\left(l^{-1}\right)\right) .
$$

For any given $j, k, p$, we define the needlet coefficients as:

$$
\begin{aligned}
\beta_{j k ; p} & :=\int_{S^{2}} T(x) \bar{\psi}_{j k ; p}(x) d x \\
= & \sqrt{\lambda_{j k}} \sum_{l \geq 1} f_{p}\left(\frac{l}{B^{j}}\right) \sum_{m=-l}^{l} a_{l m} Y_{l m}\left(\xi_{j k}\right),
\end{aligned}
$$


so that

$$
\mathbb{E}\left(\beta_{j k ; p}\right)=\sqrt{\lambda_{j k}} \sum_{l \geq 1} f_{p}\left(\frac{l}{B^{j}}\right) \sum_{m=-l}^{l} \mathbb{E}\left(a_{l m}\right) Y_{l m}\left(\xi_{j k}\right)=0 .
$$

Under Condition 2, the following result is given in [30] and [39].

Lemma 2.1. If $0<4 p+2-\alpha_{0} \leq Q$, then under Condition 2, there exists a constant $C_{Q}>0$, such that

$$
\operatorname{Corr}\left(\beta_{j k ; p}, \beta_{j^{\prime} k^{\prime} ; p}\right) \leq \frac{C_{Q}}{\left[1+B^{\left(\left(j+j^{\prime}\right) / 2-\log _{B}\left[\left(j+j^{\prime}\right) / 2\right]\right)} d\left(\xi_{j k}, \xi_{j^{\prime} k^{\prime}}\right)\right]^{\left(4 p+2-\alpha_{0}\right)}} .
$$

Assume now that from the observations over the random field, we are able to build the following set of quantities

$$
\widehat{\Lambda}_{j ; p}:=\sum_{l \geq 1} f_{p}^{2}\left(\frac{l}{B^{j}}\right)(2 l+1) \widehat{C}_{l} \simeq \sum_{k=1}^{N_{j}} \beta_{j k ; p}^{2} \text { for each } j \in\left[J_{0}, J_{L}\right]
$$

where the last approximation is motivated by the nearly tight frame property, as in 39 .

The next result describes the asymptotic behaviour of the variance-covariance matrix of $\widehat{\Lambda}_{j ; p}$ in terms of $j$.

Lemma 2.2. If Condition 1 holds with $0<4 p+2-\alpha_{0} \leq Q$, fixed $\Delta j \in \mathbb{Z}$, we have

$$
\begin{gathered}
\lim _{j \rightarrow \infty} \frac{1}{B^{2\left(1-\alpha_{0}\right) j}} \operatorname{Var}\left(\widehat{\Lambda}_{j ; p}\right)=\frac{2 G_{0}^{2}}{4^{4 p+\left(1-\alpha_{0}\right)}} \Gamma\left(4 p+1-\alpha_{0}\right) ; \\
\lim _{j \rightarrow \infty} \frac{1}{B^{2\left(1-\alpha_{0}\right) j}} \operatorname{Cov}\left(\Lambda_{j ; p}, \Lambda_{j+\Delta j ; p}\right)=2 G_{0}^{2} \frac{\tau_{B}(\Delta j)}{4^{4 p+\left(1-\alpha_{0}\right)}} \Gamma\left(4 p+1-\alpha_{0}\right),
\end{gathered}
$$

where

$$
\tau_{p}(\Delta j):=B^{\Delta j\left(1-\alpha_{0}\right)} \cosh (\Delta j \log B)^{-\left(4 p-\alpha_{0}+1\right)} .
$$

Proof. Simple calculations lead to:

$$
\begin{aligned}
\operatorname{Var}\left(\Lambda_{j ; p}\right) & =\operatorname{Var}\left(\sum_{k=1}^{N_{j}} \beta_{j k ; p}^{2}\right)=\sum_{l \geq 1} f_{p}^{4}\left(\frac{l}{B^{j}}\right)(2 l+1)^{2} \operatorname{Var}\left(\widehat{C}_{l}\right) \\
& =2 \sum_{l \geq 1} f_{p}^{4}\left(\frac{l}{B^{j}}\right)(2 l+1) C_{l}^{2},
\end{aligned}
$$

while, for $\Delta j \in \mathbb{Z}$,

$$
\operatorname{Cov}\left(\Lambda_{j ; p}, \Lambda_{j+\Delta j ; p}\right)=\operatorname{Cov}\left(\sum_{k_{1}=1}^{N_{j}} \beta_{j k_{1} ; p}^{2}, \sum_{k_{2}=1}^{N_{j+\Delta j}} \beta_{j+\Delta j k_{2} ; p}^{2}\right)
$$




$$
\begin{aligned}
& =\operatorname{Cov}\left(\sum_{l_{1} \geq 1} f_{p}^{2}\left(\frac{l_{1}}{B^{j}}\right)\left(2 l_{1}+1\right) \widehat{C}_{l_{1}}, \sum_{l_{2} \geq 1}\left(\frac{l_{2}}{B^{j+\Delta j}}\right)\left(2 l_{2}+1\right) \widehat{C}_{l_{2}}\right) \\
& =\sum_{l \geq 1} f_{p}^{2}\left(\frac{l}{B^{j}}\right) f_{p}^{2}\left(\frac{l}{B^{j+\Delta j}}\right)(2 l+1)^{2} \operatorname{Var}\left(\widehat{C}_{l}\right) \\
& =2 \sum_{l \geq 1} f_{p}^{2}\left(\frac{l}{B^{j}}\right) f_{p}^{2}\left(\frac{l}{B^{j+\Delta j}}\right)(2 l+1) C_{l}^{2} .
\end{aligned}
$$

Under Condition 1, by applying Lemma A.2, in view of the equation (A.1) with $a=4$ and $n=1-2 \alpha_{0}$, we have:

$$
\begin{aligned}
\operatorname{Var}\left(\Lambda_{j ; p}\right) & =4 G_{0}^{2} \sum_{l \geq 1} f_{p}^{4}\left(\frac{l}{B^{j}}\right)\left(l^{1-2 \alpha_{0}}+o_{l}\left(l^{1-2 \alpha_{0}}\right)\right) \\
& =2 G_{0}^{2} \frac{B^{2\left(1-\alpha_{0}\right) j}}{4^{4 p+\left(1-\alpha_{0}\right)}} \Gamma\left(4 p+1-\alpha_{0}\right)+o_{j}\left(B^{2\left(1-\alpha_{0}\right) j}\right),
\end{aligned}
$$

while, for the equation (A.2) with $a_{1}=a_{2}=2, n=1-2 \alpha_{0}$ and $\tau_{p}(\Delta j)=$ $\tau_{p, 2,2}(\Delta j)$, we obtain:

$$
\begin{gathered}
\operatorname{Cov}\left(\Lambda_{j ; p}, \Lambda_{j+\Delta j ; p}\right) \\
=4 G_{0}^{2} \sum_{l \geq 1} f_{p}^{2}\left(\frac{l}{B^{j}}\right) f_{p}^{2}\left(\frac{l}{B^{j+\Delta j}}\right) l^{1-2 \alpha_{0}}+o_{l}(1) \\
=2 G_{0}^{2} \frac{\tau_{B}(\Delta j)}{4^{4 p+\left(1-\alpha_{0}\right)}} B^{2\left(1-\alpha_{0}\right) j} \Gamma\left(4 p+1-\alpha_{0}\right)+o\left(B^{2\left(1-\alpha_{0}\right) j}\right),
\end{gathered}
$$

as claimed.

\section{Mexican Needlet Whittle-like approximation to likelihood function}

In this Section, our aim is to define a mexican needlet Whittle-like approximation to the log-likelihood function of isotropic and Gaussian random fields on the unit sphere under Condition 1 and to develop the corresponding estimators. We will follow a strategy analogue to the one used by [13, (see also $\mathbf{1 2}$ and [46]). We let

$$
\overrightarrow{\boldsymbol{\beta}}_{j ; p}=\left(\beta_{j 1 ; p}, \beta_{j 2 ; p}, \ldots, \beta_{j N_{j} ; p}\right)
$$

where $\beta_{j k ; p}$ is defined as in (2.7). Again, under the hypothesis of isotropy and Gaussianity for $T$, we have

$$
\overrightarrow{\boldsymbol{\beta}}_{j ; p} \sim N(0, \Gamma)
$$

where

$$
\Gamma=\left[\operatorname{Cov}\left(\beta_{j k ; p}, \beta_{j k^{\prime} ; p}\right)\right]_{k, k^{\prime}}=\frac{1}{N_{j}}\left(\sum_{l \geq 1} f_{p}^{2}\left(\frac{l}{B^{j}}\right)(2 l+1) C_{l}\right) I_{N_{j}},
$$

in view of (2.7) and Lemma 2.1 (see also [12, 13]). The likelihood function is then defined as

$$
\mathcal{L}\left(\vartheta ; \overrightarrow{\boldsymbol{\beta}}_{j ; p}\right)=(2 \pi)^{-N_{j}}(\operatorname{det} \Gamma)^{-1 / 2} \exp \left\{-\frac{1}{2} \overrightarrow{\boldsymbol{\beta}}_{j ; p}^{T} \Gamma^{-1} \vec{\beta}_{j ; p}\right\}
$$

Let 


$$
K_{j}^{M}(\alpha):=\frac{1}{N_{j}} \sum_{l \geq 1} f_{p}^{2}\left(\frac{l}{B^{j}}\right)(2 l+1) l^{-\alpha} .
$$

Under Condition 1 we have:

$$
\mathcal{L}\left(\alpha, G ; \overrightarrow{\boldsymbol{\beta}}_{j ; p}\right)=(2 \pi)^{-N_{j}}\left(G K_{j}^{M}(\alpha)\right)^{-N_{j} / 2} \exp \left\{-\frac{1}{2} \frac{\sum_{k} \beta_{j k ; p}^{2}}{G K_{j}^{M}(\alpha)}\right\},
$$

and the corresponding approximate log-likelihood is

$$
-2 \log \mathcal{L}\left(\alpha, G ; \overrightarrow{\boldsymbol{\beta}}_{j ; p}\right)=\sum_{k}\left\{\frac{\beta_{j k ; p}^{2}}{G K_{j}^{M}(\alpha)}-\log \left(\frac{\beta_{j k ; p}^{2}}{G K_{j}^{M}(\alpha)}\right)\right\},
$$

up to an additive constant.

By summing with respect to $j$, we obtain.

$$
R_{J_{0}, J_{L}}^{M}(\alpha, G):=\left(\sum_{j=J_{0}}^{J_{L}} N_{j}\right)^{-1} \sum_{j=J_{0}}^{J_{L}}-2 \log \mathcal{L}\left(\alpha, G ; \boldsymbol{\beta}_{j ; p}\right),
$$

where the choice for $J_{0}, J_{L}$ will be discussed later. Hence we define (cfr. $1 \mathbf{1 2}$ and [13])

$$
\left(\widehat{\alpha}_{J_{0}, J_{L}}^{M}, \widehat{G}_{J_{0}, J_{L}}^{M}\right)=\arg \min _{(\alpha, G) \in \Theta} R_{J_{0}, J_{L}}^{M}(\alpha, G),
$$

where $\Theta=[2,+\infty) \times(0,+\infty)$. Computing the derivative of $R_{J_{0}, J_{L}}^{M}$ with respect to $G$ and setting it equal to zero, we have

$$
0=\frac{\partial}{\partial G} R_{J_{0}, J_{L}}^{M}(\alpha, G)=\frac{1}{\sum_{j=J_{0}}^{J_{L}} N_{j}} \sum_{j=J_{0}}^{J_{L}}\left[-\frac{\sum_{k} \beta_{j k ; p}^{2}}{G^{2} K_{j}^{M}(\alpha)}+\frac{N_{j}}{G}\right],
$$

whence

$$
\widehat{G}_{J_{0}, J_{L}}^{M}=\widehat{G}_{J_{0}, J_{L}}^{M}(\alpha)=\frac{1}{\sum_{j=J_{0}}^{J_{L}} N_{j}} \sum_{j=J_{0}}^{J_{L}} \frac{\sum_{k} \beta_{j k ; p}^{2}}{K_{j}^{M}(\alpha)}=\frac{1}{\sum_{j=J_{0}}^{J_{L}} N_{j}} \sum_{j=J_{0}}^{J_{L}} \frac{\Lambda_{j ; p}}{K_{j}^{M}(\alpha)} .
$$

Since

$$
\begin{gathered}
\left.\frac{\partial^{2}}{\partial G^{2}} R_{J_{0}, J_{L}}^{M}(\alpha, G)\right|_{G=\widehat{G}_{J_{0}, J_{L}}^{M}(\alpha)} \\
=\left.\frac{1}{\sum_{j=J_{0}}^{J_{L}} N_{j}} \sum_{j=J_{0}}^{J_{L}} \frac{1}{G^{2}}\left(\frac{2 \Lambda_{j ; p}}{G K_{j}^{M}(\alpha)}-N_{j}\right)\right|_{G=\widehat{G}_{J_{0}, J_{L}}^{M}(\alpha)}=\frac{1}{\left(\widehat{G}_{J_{0}, J_{L}}^{M}(\alpha)\right)^{2}}>0,
\end{gathered}
$$

and $R_{J_{0}, J_{L}}^{M}(\alpha, G) \rightarrow+\infty$ as $G \rightarrow 0$ or $\infty$, the second derivative test yields that $R_{J_{0}, J_{L}}^{M}(\alpha, G)$ has a unique minimum over the domain on $\widehat{G}_{J_{0}, J_{L}}^{M}(\alpha)$. Therefore, we can define

$$
\begin{aligned}
R_{J_{0}, J_{L}}^{M}(\alpha): & =R_{J_{0}, J_{L}}^{M}\left(\alpha, \widehat{G}_{J_{0}, J_{L}}^{M}(\alpha)\right) \\
= & 1+\log \widehat{G}_{J_{0}, J_{L}}^{M}(\alpha)-\frac{1}{\sum_{j=J_{0}}^{J_{L}} N_{j}} \sum_{j=J_{0}}^{J_{L}} \sum_{k} \log \frac{\beta_{j k ; p}^{2}}{K_{j}^{M}(\alpha)} .
\end{aligned}
$$


REMARK 3.1. In this formula it is necessary to fix explicitly the values of $L, J_{0}$ and $J_{L}$. Let us fix $L$ as the highest multipole level available from the dataset. Given $L$, as stressed above, differently from the standard needlet case (see for instance [40, [41), in the mexican needlet case the weight function does not have a compact support. Therefore, for computational reasons, we must find a criterion to choose the suitable extrema for the sums over $j$ involved. Considering (see again [21]) the behaviour of $f_{p}(\cdot)$, we can fix thresholds $\varepsilon_{B, 1}(L), \varepsilon_{B, 2}(L)$, such that:

$$
\begin{aligned}
& J_{0}=\max \left\{j \in \mathbb{Z}: f_{p}\left(\frac{1}{B^{j+1}}\right)>\varepsilon_{B, 1}(L) f_{p}\left(\frac{1}{B^{j}}\right)\right\}, \\
& J_{L}=\min \left\{j \in \mathbb{Z}: f_{p}\left(\frac{L}{B^{j}}\right)<\varepsilon_{B, 2}(L) f_{p}\left(\frac{L}{B^{j-1}}\right)\right\} .
\end{aligned}
$$

If, for instance, we choose,

$$
\varepsilon_{B, 1}(L)=\frac{1}{B^{2 p}} \exp \left(\frac{B-1}{B^{2}}\right), \varepsilon_{B, 2}(L)=\frac{1}{B^{2 p}} \exp \left(B^{2}\left(B^{2}-1\right)\right)
$$

we find $B^{J_{0}}=B, B^{J_{L}}=L / B$, similarly to the classical needlet case as described in 13 .

\section{Asymptotic Properties}

In this Section, we prove weak consistency for the estimators $\widehat{\alpha}_{J_{0}, J_{L}}^{M}$ and $\widehat{G}_{J_{0}, J_{L}}^{M}$, and for the former also asymptotic Gaussianity. We begin with some definitions: let

$$
G_{J_{0} ; J_{L}}^{M}(\alpha)=\frac{1}{\sum_{j=J_{0}}^{J_{L}} N_{j}} \sum_{j=J_{0}}^{J_{L}} N_{j} \frac{G_{0} K_{j}^{M}\left(\alpha_{0}\right)}{K_{j}^{M}(\alpha)} .
$$

Computing the first and second order derivatives of $G_{J_{0} ; J_{L}}^{M}(\alpha)$, indexed by $n$, we obtain

$$
\begin{aligned}
G_{J_{0} ; J_{L}, n}^{M}(\alpha) & :=\frac{d^{n}}{d \alpha^{n}} G_{J_{0} ; J_{L}}^{M}(\alpha) \\
& =\frac{G_{0}}{\sum_{j=J_{0}}^{J_{L}} N_{j}} \sum_{j=J_{0}}^{J_{L}} N_{j} \frac{K_{j}^{M}\left(\alpha_{0}\right)}{K_{j}^{M}(\alpha)} U_{j ; n}(\alpha),
\end{aligned}
$$

where (see Proposition 1) in the Appendix, we have

$$
\begin{gathered}
U_{j ; 1}(\alpha)=\left(-\frac{K_{j, 1}^{M}(\alpha)}{K_{j}^{M}(\alpha)}\right)=\left(j \log B+\frac{I_{p, 1}(\alpha)}{I_{p, 0}(\alpha)}+o_{j}(1)\right), \\
U_{j ; 2}(\alpha)=2\left(\frac{K_{j, 1}^{M}(\alpha)}{K_{j}^{M}(\alpha)}\right)^{2}-\frac{K_{j, 2}^{M}(\alpha)}{K_{j}^{M}(\alpha)} \\
=j^{2} \log ^{2} B+2 j \log B \frac{I_{p, 1}(\alpha)}{I_{p, 0}(\alpha)}+2\left(\frac{I_{p, 1}(\alpha)}{I_{p, 0}(\alpha)}\right)^{2}-\frac{I_{p, 2}(\alpha)}{I_{p, 0}(\alpha)}+o_{j}(1),
\end{gathered}
$$

Furthermore, we fix

$$
U_{j ; 0}(\alpha)=1, G_{J_{0} ; J_{L}, 0}^{M}(\alpha)=G_{J_{0} ; J_{L}}^{M}(\alpha),
$$


(since now, we will use either $G_{J_{0} ; J_{L}, 0}^{M}(\alpha)$ or $G_{J_{0} ; J_{L}}^{M}(\alpha)$ ). Recalling that $N_{j}=$ $C_{B} B^{2 j}$. Thus by (A.6), we have for $s=0,1,2$,

$$
\begin{aligned}
G_{J_{0} ; J_{L}, s}^{M}(\alpha) & =\frac{G_{0}}{\sum_{j=J_{0}}^{J_{L}} N_{j}} \sum_{j=J_{0}}^{J_{L}} N_{j} \frac{K_{j}^{M}\left(\alpha_{0}\right)}{K_{j}^{M}(\alpha)} U_{j ; s}(\alpha) \\
& =G_{0} \frac{(p+1)^{-\frac{\alpha-\alpha_{0}}{2}}}{\sum_{j=J_{0}}^{J_{L}} B^{2 j}} \sum_{j=J_{0}}^{J_{L}} B^{\left(2+\alpha-\alpha_{0}\right) j} U_{j ; s}(\alpha) .
\end{aligned}
$$

The next auxiliary result is as follows:

Lemma 4.1. Assume Condition 1 holds with $0<4 p+2-\alpha_{0} \leq Q$. We have that

$$
\begin{gathered}
\lim _{J_{L} \rightarrow \infty} \mathbb{E}\left(\widehat{G}_{J_{0}, J_{L}}^{M}\left(\alpha_{0}\right)\right) \rightarrow G_{0}, \\
\lim \frac{1}{B^{2 J_{L}}} \operatorname{Var}\left(\frac{\widehat{G}_{J_{0}, J_{L}}^{M}\left(\alpha_{0}\right)}{G_{0}}\right)=\frac{B^{2}-1}{B^{2}} \sigma_{0}^{2}\left(1+\widetilde{\tau}_{0}\right),
\end{gathered}
$$

where

$$
\sigma_{0}^{2}:=\sigma_{0}^{2}\left(p, \alpha_{0}\right)=\frac{2}{2^{4 p-\alpha_{0}}} \frac{\Gamma\left(4 p+1-\alpha_{0}\right)}{\Gamma^{2}\left(2 p-\frac{\alpha_{0}}{2}+1\right)},
$$

and $\widetilde{\tau}_{0}$ is as defined in Lemma B.1.

Proof. We have

$$
\begin{aligned}
\mathbb{E}\left(\widehat{G}_{J_{0}, J_{L}}^{M},\left(\alpha_{0}\right)\right) & =\frac{1}{\sum_{j=J_{0}}^{J_{L}} N_{j}} \sum_{j=J_{0}}^{J_{L}} \frac{\mathbb{E}\left(\widehat{\Lambda}_{j}\right)}{K_{j}^{M}(\alpha)} \\
& =\frac{G_{0}}{\sum_{j=J_{0}}^{J_{L}} N_{j}} \sum_{j=J_{0}}^{J_{L}} \frac{\sum_{l} f_{p}^{2}\left(\frac{l}{B^{j}}\right)(2 l+1) l^{-\alpha_{0}}\left(1+O\left(l^{-1}\right)\right)}{K_{j}^{M}(\alpha)} \\
& =G_{0}+o_{J_{L}}(1) .
\end{aligned}
$$

On the other hand, we prove that

$$
\operatorname{Cov}\left(\frac{\Lambda_{j ; p}}{G_{0} K_{j}^{M}(\alpha)}, \frac{\Lambda_{j+\Delta j ; p}}{G_{0} K_{j+\Delta j}^{M}(\alpha)}\right)=c_{B}^{2} \sigma_{0}^{2} B^{2 j} B^{\alpha_{0} \Delta j} \tau_{B}(\Delta j) .
$$

We can indeed observe from Theorem 2.2 that

$$
\begin{gathered}
\operatorname{Cov}\left(\frac{\widehat{\Lambda}_{j}}{G_{0} K_{j}^{M}(\alpha)}, \frac{\widehat{\Lambda}_{j+\Delta j}}{G_{0} K_{j+\Delta j}^{M}(\alpha)}\right) \\
=\frac{B^{\alpha \Delta j}}{G_{0}^{2} I_{p, 0}^{2}(\alpha) B^{-2 \alpha j}} \operatorname{Cov}\left(\widehat{\Lambda}_{j}, \widehat{\Lambda}_{j+\Delta j}\right) \\
=\frac{B^{\alpha \Delta j}}{I_{p, 0}^{2}(\alpha)} \frac{2 \Gamma\left(4 p+1-\alpha_{0}\right)}{4^{4 p+1-\alpha_{0}}} \tau_{B}(\Delta j) B^{2\left(1+\alpha-\alpha_{0}\right) j} \\
=\frac{2 c_{B}^{2}}{2^{4 p-2 \alpha_{0}+\alpha}} \frac{\Gamma\left(4 p+1-\alpha_{0}\right)}{\Gamma^{2}\left(2 p-\frac{\alpha}{2}+1\right)} \tau_{B}(\Delta j) B^{\alpha \Delta j} B^{2\left(1+\alpha-\alpha_{0}\right) j} .
\end{gathered}
$$


Hence

$$
\begin{aligned}
& \operatorname{Var}\left(\frac{\widehat{G}_{J_{0}, J_{L}}^{M}(\alpha)}{G_{0}}\right) \\
= & \frac{1}{\left(\sum_{j=J_{0}}^{J_{L}} N_{j}\right)^{2}} \operatorname{Cov}\left(\sum_{j=J_{0}}^{J_{L}} \frac{\Lambda_{j ; p}}{G_{0} K_{j}^{M}(\alpha)}, \sum_{\Delta j=J_{0}-j}^{J_{L}-j} \frac{\Lambda_{j+\Delta j ; p}}{G_{0} K_{j+\Delta j}^{M}(\alpha)}\right) \\
= & \frac{1}{\left(\sum_{j=J_{0}}^{J_{L}} N_{j}\right)^{2}} \sum_{j=J_{0}}^{J_{L}} \sum_{j=J_{0}-j}^{J_{L}-j} \operatorname{Cov}^{2}\left(\frac{\sum_{k_{1}} \beta_{j k_{1} ; p}^{2}}{G_{0} K_{j}^{M}(\alpha)}, \frac{\sum_{k_{2}} \beta_{j+\Delta j, k ; p}^{2}}{G_{0} K_{j+\Delta j}^{M}(\alpha)}\right) \\
= & \frac{1}{\left(\sum_{j=J_{0}}^{J_{L}} B^{2 j}\right)^{2}} \frac{\Gamma\left(4 p+1-\alpha_{0}\right)}{4^{2 p-\alpha_{0}+\frac{\alpha}{2}}} \frac{J_{L}}{\Gamma^{2}\left(2 p-\frac{\alpha}{2}+1\right)} \sum_{j=J_{0}} B^{2(1+\alpha-\alpha) j} \sum_{\Delta j=J_{0}-j}^{J_{L}-j} \tau_{B}(\Delta j) B^{\alpha \Delta j} .
\end{aligned}
$$

Following Lemmas B.1 and 2, and computing in $\alpha=\alpha_{0}$, we have

$$
\begin{aligned}
\operatorname{Var}\left(\frac{\widehat{G}_{J_{0}, J_{L}}^{M}\left(\alpha_{0}\right)}{G_{0}}\right) & =\frac{2\left(1+\widetilde{\tau}_{0}\right)}{2^{4 p-\alpha_{0}}} \frac{\Gamma\left(4 p+1-\alpha_{0}\right)}{\Gamma^{2}\left(2 p-\frac{\alpha_{0}}{2}+1\right)}\left(\sum_{j=J_{0}}^{J_{L}} B^{2 j}\right)^{-1} \\
& =\frac{B^{2}-1}{B^{2}} \sigma_{0}^{2}\left(1+\widetilde{\tau}_{0}\right) B^{-2 J_{L}}+o\left(B^{-2 J_{L}}\right)
\end{aligned}
$$

Lemma 4.2. Under Condition 1, we have for $s=0,1,2$ :

$$
\sup \left|\frac{\widehat{G}_{J_{0}, J_{L} ; s}^{M}(\alpha)}{G_{J_{0}, J_{L} ; s}^{M}(\alpha)}\right| \rightarrow_{p} 0
$$

Proof. Under Condition 1, we can readily obtain that

$$
\begin{gathered}
\frac{\widehat{G}_{J_{0}, J_{L}, s}^{M}(\alpha)}{G_{J_{0}, J_{L}, s}^{M}(\alpha)}-1=\frac{\sum_{j=J_{0}}^{J_{L}} \frac{\sum_{k} \beta_{j k ; p}^{2}}{K_{j}^{M}(\alpha)} U_{j ; s}(\alpha)}{\sum_{j=J_{0}}^{J_{L}} N_{j} \frac{G_{0} K_{j}^{M}\left(\alpha_{0}\right)}{K_{j}^{M}(\alpha)} U_{j ; s}(\alpha)}-1 \\
=\frac{\sum_{j=J_{0}}^{J_{L}} \sqrt{N_{j}} \frac{K_{j}^{M}\left(\alpha_{0}\right)}{K_{j}^{M}(\alpha)} U_{j ; s}(\alpha)\left(\frac{1}{\sqrt{N_{j}}} \sum_{k}\left(\frac{\beta_{j k ; p}^{2}}{G_{0} K_{j}^{M}\left(\alpha_{0}\right)}-1\right)\right)}{\sum_{j=J_{0}}^{J_{L}} N_{j} \frac{K_{j}^{M}\left(\alpha_{0}\right)}{K_{j}^{M}(\alpha)} U_{j ; s}(\alpha)},
\end{gathered}
$$


so that

$$
\begin{aligned}
& \mathbb{P}\left(\left|\frac{\sum_{j=J_{0}}^{J_{L}} \sqrt{N_{j}} \frac{K_{j}^{M}\left(\alpha_{0}\right)}{K_{j}^{M M}(\alpha)} U_{j ; s}(\alpha)\left(\frac{1}{\sqrt{N_{j}}} \sum_{k}\left(\frac{\beta_{j k ; p}^{2}}{\sum_{0} K_{j}^{M}\left(\alpha_{0}\right)}-1\right)\right)}{\sum_{j=J_{0}}^{J_{L}} N_{j} \frac{K_{j}^{M}\left(\alpha_{0}\right)}{K_{j}^{M}(\alpha)} U_{j ; s}(\alpha)}\right|>\delta_{\varepsilon}\right) \\
\leq & \mathbb{P}\left(\left(J_{L}+J_{0}+1\right)\left|\frac{\sum_{j=J_{0}}^{J_{L}} \sqrt{N_{j}} \frac{K_{j}^{M}\left(\alpha_{0}\right)}{K_{j}^{M}(\alpha)} U_{j ; s}(\alpha) \mid}{\sum_{j=J_{0}}^{J_{L}} N_{j} \frac{K_{j}^{M}\left(\alpha_{0}\right)}{K_{j}^{M}(\alpha)} U_{j ; s}(\alpha)}\right|\right. \\
& \left.\times \frac{\sup _{j}\left(\frac{1}{\sqrt{N_{j}}} \sum_{k}\left(\frac{\beta_{j k ; p}^{2}}{G_{0} K_{j}^{M M}\left(\alpha_{0}\right)}-1\right)\right)}{\left(J_{L}+J_{0}+1\right)}>\delta_{\varepsilon}\right) .
\end{aligned}
$$

In view of (A.4) and (A.5), we obtain

$$
\begin{gathered}
\frac{\sum_{j=J_{0}}^{J_{L}} \sqrt{N_{j}} \frac{K_{j}^{M}\left(\alpha_{0}\right)}{K_{j}^{M}(\alpha)} U_{j ; s}(\alpha)}{\sum_{j=J_{0}}^{J_{L}} N_{j} \frac{K_{j}^{M}\left(\alpha_{0}\right)}{K_{j}^{M}(\alpha)} U_{j ; s}(\alpha)} \\
=\frac{\sum_{j=J_{0}}^{J_{L}} B^{j\left(1+\alpha-\alpha_{0}\right)} j^{s}}{\sum_{j=J_{0}}^{J_{L}} B^{j\left(2+\alpha-\alpha_{0}\right)} j^{s}} \\
=\frac{B^{\left(2+\alpha-\alpha_{0}\right)}-1}{B\left(B^{\left(1+\alpha-\alpha_{0}\right)}-1\right)} \frac{J_{L} B^{J_{L}\left(1+\alpha-\alpha_{0}\right)}-J_{0} B^{J_{0}\left(1+\alpha-\alpha_{0}\right)-1}}{J_{L} B^{J_{L}\left(2+\alpha-\alpha_{0}\right)}-J_{0} B^{J_{0}\left(2+\alpha-\alpha_{0}\right)-1}} \\
=O\left(B^{-J_{L}}\right)
\end{gathered}
$$

so that

$$
\sup _{j}\left|\left(J_{L}+J_{0}+1\right) \frac{\sum_{j=J_{0}}^{J_{L}} \sqrt{N_{j}} \frac{K_{j}^{M}\left(\alpha_{0}\right)}{K_{j}^{M}(\alpha)} U_{j ; s}(\alpha)}{\sum_{j=J_{0}}^{J_{L}} N_{j} \frac{K_{j}^{M}\left(\alpha_{0}\right)}{K_{j}^{M}(\alpha)} U_{j ; s}(\alpha)}\right|<+\infty .
$$

On the other hand, we have by Chebyshev's inequality and Lemma 4.1 that

$$
\begin{aligned}
& \mathbb{P}\left(\left|\left(\frac{1}{\sqrt{N_{j}}} \sum_{k}\left(\frac{\beta_{j k ; p}^{2}}{G_{0} K_{j}^{M}\left(\alpha_{0}\right)}-1\right)\right)\right|>\delta_{\varepsilon}\left(J_{L}+J_{0}+1\right)^{2}\right) \\
& \leq \frac{1}{\delta_{\varepsilon}^{2}\left(J_{L}+J_{0}+1\right)^{2}} \operatorname{Var}\left(\frac{1}{\sqrt{N_{j}}} \sum_{k}\left(\frac{\beta_{j k ; p}^{2}}{G_{0} K_{j}^{M}\left(\alpha_{0}\right)}-1\right)\right) \\
& =O\left(\frac{1}{\left(J_{L}+J_{0}+1\right)^{2}}\right)
\end{aligned}
$$

whence

$$
\begin{gathered}
\mathbb{P}\left(\sup _{j=J_{0}, \ldots J_{L}}\left|\left(\frac{1}{\sqrt{N_{j}}} \sum_{k}\left(\frac{\beta_{j k ; p}^{2}}{G_{0} K_{j}^{M}\left(\alpha_{0}\right)}-1\right)\right)\right|>\delta_{\varepsilon}\left(J_{L}+J_{0}+1\right)^{2}\right) \\
\leq\left(J_{L}+J_{0}+1\right) \sup _{j=J_{0}, \ldots J_{L}} \mathbb{P}\left(\left|\left(\frac{1}{\sqrt{N_{j}}} \sum_{k}\left(\frac{\beta_{j k ; p}^{2}}{G_{0} K_{j}^{M}\left(\alpha_{0}\right)}-1\right)\right)\right|>\delta_{\varepsilon}\left(J_{L}+J_{0}+1\right)^{2}\right)
\end{gathered}
$$




$$
\leq O\left(\frac{1}{\left(J_{L}+J_{0}+1\right)}\right)
$$

Let us focus now our attention on consistency, following a technique developed in [7] and used in [46] for long memory processes (see also [12] and [13).

Theorem 4.3. Assume Condition 1 holds with $0<4 p+2-\alpha_{0} \leq Q$, we have, as $J_{L} \rightarrow \infty$,

$$
\begin{aligned}
\widehat{\alpha}_{J_{0}, J_{L}}^{M} & \rightarrow{ }^{2} \alpha_{0}, \\
\widehat{G}_{J_{0}, J_{L}}^{M} & \rightarrow{ }^{M} G_{0} .
\end{aligned}
$$

Proof. Following [46] (see also [13, for the standard needlet case), we let

$$
\begin{aligned}
\Delta R_{J_{0}, J_{L}}^{M}\left(\alpha, \alpha_{0}\right)= & R_{J_{0}, J_{L}}^{M}(\alpha)-R_{J_{0}, J_{L}}^{M}\left(\alpha_{0}\right) \\
= & \log \frac{\widehat{G}_{J_{0}, J_{L}}^{M}(\alpha)}{G_{J_{0}, J_{L}}^{M}(\alpha)}-\log \frac{\widehat{G}_{J_{0}, J_{L}}^{M}\left(\alpha_{0}\right)}{G_{0}} \\
& +\log \frac{G_{J_{0}, J_{L}}^{M}(\alpha)}{G_{0}}+\frac{1}{\sum_{j=J_{0}}^{J_{L}} N_{j}} \sum_{j=J_{0}}^{J_{L}} N_{j} \log \frac{K_{j}^{M}(\alpha)}{K_{j}^{M}\left(\alpha_{0}\right)} \\
= & U_{J_{0}, J_{L}}^{M}(\alpha)-T_{J_{0}, J_{L}}^{M}(\alpha),
\end{aligned}
$$

where

$$
\begin{aligned}
T_{J_{0}, J_{L}}^{M}(\alpha) & =\log \frac{\widehat{G}_{J_{0}, J_{L}}^{M}(\alpha)}{G_{J_{0}, J_{L}}^{M}(\alpha)}-\log \frac{\widehat{G}_{J_{0}, J_{L}}^{M}\left(\alpha_{0}\right)}{G_{0}} \\
U_{J_{0}, J_{L}}^{M}(\alpha) & =\log \frac{G_{J_{0}, J_{L}}^{M}(\alpha)}{G_{0}}+\frac{1}{\sum_{j=J_{0}}^{J_{L}} N_{j}} \sum_{j=J_{0}}^{J_{L}} N_{j} \log \frac{K_{j}^{M}(\alpha)}{K_{j}^{M}\left(\alpha_{0}\right)} .
\end{aligned}
$$

For any $\varepsilon>0$, we have

$$
\begin{aligned}
\mathbb{P}\left(\left|\widehat{\alpha}_{J_{0}, J_{L}}^{M}-\alpha_{0}\right|>\varepsilon\right) & =\mathbb{P}\left(\min _{\left|\alpha-\alpha_{0}\right|>\varepsilon} \Delta R_{J_{0}, J_{L}}^{M}\left(\alpha, \alpha_{0}\right) \leq 0\right) \\
& =\mathbb{P}\left(\min _{\left|\alpha-\alpha_{0}\right|>\varepsilon} T_{J_{0}, J_{L}}^{M}(\alpha)+U_{J_{0}, J_{L}}^{M}(\alpha) \leq 0\right) .
\end{aligned}
$$

Hence, by combining Lemmas 4.4 and 4.5 , we obtain

$$
\begin{gathered}
\lim _{J_{L} \rightarrow+\infty} U_{J_{0}, J_{L}}^{M}\left(\alpha, \alpha_{0}\right)>0, \\
\sup _{\alpha}\left|T_{J_{0}, J_{L}}^{M}\left(\alpha, \alpha_{0}\right)\right|=o_{p}(1),
\end{gathered}
$$

as claimed.

Lemma 4.4. Let $U_{J_{0}, J_{L}}^{M}\left(\alpha, \alpha_{0}\right)$ be defined as in (4.6). For all $\varepsilon<\alpha_{0}-\alpha<2$

$$
=\lim _{J_{L} \rightarrow+\infty}\left(\log \frac{1}{\sum_{J_{L} \rightarrow+\infty}^{J_{L}} N_{j}} \sum_{j=J_{0}}^{J_{L}} N_{j} \frac{K_{j}^{M}\left(\alpha_{0}\right)}{K_{j}^{M}(\alpha)}-\frac{1}{\sum_{j=J_{0}}^{J_{L}} N_{j}} \sum_{j=J_{0}}^{J_{L}} N_{j} \log \frac{K_{j}^{M}\left(\alpha_{0}\right)}{K_{j}^{M}(\alpha)}\right)
$$




$$
=\log \frac{B^{2}-1}{B^{\left(2+\alpha-\alpha_{0}\right)}-1}+\log B \frac{B^{2}}{B^{2}-1} \alpha-\alpha_{0}>\delta_{\varepsilon}>0 .
$$

if $\alpha_{0}-\alpha=2$ we have

$$
\lim _{J_{L} \rightarrow+\infty} \frac{1}{\log J_{L}} U_{J_{0}, J_{L}}\left(\alpha, \alpha_{0}\right)=1
$$

and if $\alpha_{0}-\alpha>2$ we have

$$
\lim _{J_{L} \rightarrow+\infty} \frac{1}{\log B^{J_{L}}} U_{J_{0}, J_{L}}\left(\alpha, \alpha_{0}\right)=\frac{\alpha_{0}-\alpha}{2}-1
$$

Proof. Consider first the case $\varepsilon<\alpha_{0}-\alpha<2$. For the sake of simplicity, we fix $J_{0}=-J_{L}$. We have that

$$
\begin{gathered}
\frac{1}{\sum_{j=-J_{l}}^{J_{L}} N_{j}} \sum_{j=-J_{L}}^{J_{L}} N_{j} \log \frac{K_{j}^{M}\left(\alpha_{0}\right)}{K_{j}^{M}(\alpha)} \\
=\frac{1}{\sum_{j=-J_{L}}^{J_{L}} N_{j}} \sum_{j=-J_{L}}^{J_{L}} N_{j}\left(\log B^{\left(\alpha-\alpha_{0}\right) j} I_{p}\left(B, \alpha-\alpha_{0}\right)+o(j)\right) \\
=\left(\alpha-\alpha_{0}\right) \log B\left(J_{L}-\frac{1}{B^{2}-1}\right)+\log \left(I_{p}\left(B, \alpha-\alpha_{0}\right)\right)+o_{J_{L}}(1) .
\end{gathered}
$$

On the other hand, we have

$$
\begin{gathered}
\log \frac{1}{\sum_{j=-J_{L}}^{J_{L}} B^{2 j}} \sum_{j=-J_{L}}^{J_{L}} N_{j} \frac{K_{j}^{M}\left(\alpha_{0}\right)}{K_{j}^{M}(\alpha)} \\
=\log \frac{I_{p}\left(B, \alpha-\alpha_{0}\right)}{\sum_{j=-J_{L}}^{J_{L}} B^{2 j}} \sum_{j=-J_{L}}^{J_{L}} B^{2 j} B^{\left(\alpha-\alpha_{0}\right) j}+o_{J_{L}}(1) \\
=\log \frac{B^{2}-1}{B^{2+\left(\alpha-\alpha_{0}\right)}-1} B^{\left(\alpha-\alpha_{0}\right)\left(J_{L}+1\right)}+\log \left(I_{p}\left(B, \alpha-\alpha_{0}\right)\right)+o_{J_{L}}(1) \\
=\log \frac{B^{2}-1}{B^{2+\left(\alpha-\alpha_{0}\right)}-1}+\left(\alpha-\alpha_{0}\right)\left(J_{L}+1\right) \log B+\log \left(I_{p}\left(B, \alpha-\alpha_{0}\right)\right) .
\end{gathered}
$$

As shown in $\mathbf{1 3}$, we have that the function

$$
l(x):=\frac{B^{2}-1}{B^{2+x}-1}+x\left(\frac{B^{2} \log B}{B^{2}-1}\right)
$$

has a unique minimum 0 at $x=0$. Therefore, for any $\left|\alpha-\alpha_{0}\right|>\varepsilon>0$, there exists a constant $\delta_{\varepsilon}>0$, such that

$$
U_{J_{0}, J_{L}}\left(\alpha, \alpha_{0}\right)>\delta_{\varepsilon}
$$

If $\alpha-\alpha_{0}<-2$, we have

$$
\begin{aligned}
\frac{1}{\log B^{2 J_{L}}} U_{J_{0}, J_{L}}\left(\alpha, \alpha_{0}\right) & \frac{1}{\log B^{2 J_{L}}}\left\{\log \left[\sum_{j=J_{0}}^{J_{L}} B^{j\left(2+\alpha-\alpha_{0}\right)}\right]-\log B^{2 J_{L}}-\frac{\left(\alpha-\alpha_{0}\right)}{\sum_{j=J_{0}}^{J_{L}} N_{j}} \sum_{j=J_{0}}^{J_{L}} N_{j} \log \frac{K_{j}^{M}\left(\alpha_{0}\right)}{K_{j}^{M}(\alpha)}\right\}+o_{J_{L}}(1) \\
= & \frac{\alpha_{0}-\alpha}{2}-1 .
\end{aligned}
$$


Finally, we have for $\alpha-\alpha_{0}=-2$

$$
\begin{gathered}
\lim _{J_{L} \rightarrow \infty} \frac{1}{\log J_{L}} U_{J_{0}, J_{L}}\left(\alpha, \alpha_{0}\right) \\
\lim _{J_{L} \rightarrow \infty} \frac{1}{\log J_{L}}\left\{-\log B^{2 J_{L}}+\log J_{L}+O_{J_{L}}(1)+\log B^{2 J_{L}} O_{J_{L}}(1)\right\}=1 .
\end{gathered}
$$

Lemma 4.5. As $J_{L} \rightarrow+\infty$, we have

$$
\sup _{\alpha}\left|T_{J_{0}, J_{L}}^{M}\left(\alpha, \alpha_{0}\right)\right|=o_{p}(1) \text {. }
$$

Proof. Because

$$
\frac{\widehat{G}_{J_{0}, J_{L}}^{M}(\alpha)}{G_{J_{0}, J_{L}}^{M}(\alpha)}=\frac{1}{G_{0}} \frac{\sum_{j=J_{0}}^{J_{L}} \frac{\Lambda_{j ; p}}{K_{j}^{M}(\alpha)}}{\sum_{j=J_{0}}^{J_{L}} N_{j} \frac{K_{j}^{M}\left(\alpha_{0}\right)}{K_{j}^{M}(\alpha)}},
$$

it follows from Lemma 4.1 that

$$
\mathbb{E}\left(\frac{\widehat{G}_{J_{0}, J_{L}}^{M}(\alpha)}{G_{J_{0}, J_{L}}^{M}(\alpha)}-1\right)=0,
$$

while

$$
\operatorname{Var}\left(\frac{\widehat{G}_{J_{0}, J_{L}}^{M}(\alpha)}{G_{J_{0}, J_{L}}^{M}(\alpha)}-1\right)=O\left(B^{-2 J_{L}}\right)
$$

Indeed, we have

$$
\begin{gathered}
\operatorname{Var}\left(\frac{\widehat{G}_{J_{0}, J_{L}}^{M}(\alpha)}{G_{J_{0}, J_{L}}^{M}(\alpha)}\right)=\left(G_{J_{0}, J_{L}}^{M}(\alpha)\right)^{-2} \operatorname{Var}\left(\widehat{G}_{J_{0}, J_{L}}^{M}(\alpha)\right) \\
=\frac{\left(B^{\left(2+\alpha-\alpha_{0}\right)}-1\right)^{2}}{\left(B^{2\left(1+\alpha-\alpha_{0}\right)}-1\right)} \frac{I_{p}\left(B, \alpha-\alpha_{0}\right)^{2}}{B^{\alpha_{0}-\alpha} 4^{2 p-\alpha_{0}+\frac{\alpha}{2}}} \frac{\Gamma\left(4 p+1-\alpha_{0}\right)}{\Gamma^{2}\left(2 p-\frac{\alpha}{2}+1\right)} B^{-2 J_{L}}+o_{J_{L}}\left(B^{-2 J_{L}}\right)
\end{gathered}
$$

By Chebyshev's inequality we have

$$
\frac{\widehat{G}_{J_{0}, J_{L}}^{M}(\alpha)}{G_{J_{0}, J_{L}}^{M}(\alpha)}-1 \rightarrow_{p} 0
$$

and from Slutsky's Lemma

$$
\log \left(\frac{\widehat{G}_{J_{0}, J_{L}}^{M}(\alpha)}{G_{J_{0}, J_{L}}^{M}(\alpha)}-1\right) \rightarrow_{p} 0 .
$$

On the other hand, by Lemma 4.2

$$
\sup \left|\frac{\widehat{G}_{J_{0}, J_{L}}^{M}(\alpha)}{G_{J_{0}, J_{L}}^{M}(\alpha)}-1\right| \rightarrow_{p} 0,
$$

as we claimed.

Our purpose now is to study an asymptotic convergence of estimator $\widehat{\alpha}_{J_{0}, J_{L}}^{M}$. 
Theorem 4.6. Let $0<4 p-\alpha_{0} \leq Q$. Assume Condition 1 holds with. Hence we have

$$
B^{J_{L}}\left(\widehat{\alpha}_{J}^{M}-\alpha_{0}\right)=O_{p}(1) \text {, as } J_{L} \rightarrow \infty .
$$

Under Condition 3, we have

$$
B^{J_{L}}\left(\widehat{\alpha}_{J}^{M}-\alpha_{0}\right) \rightarrow_{p}-\frac{I_{p, 0}\left(\alpha_{0}+1\right)}{I_{p, 0}\left(\alpha_{0}\right)} \frac{\log B}{(B+1)} \kappa .
$$

Under Condition 4, we have

$$
B^{J_{L}}\left(\widehat{\alpha}_{J}^{M}-\alpha_{0}\right) \rightarrow_{d} N\left(0, \varsigma_{0}^{2}\right)
$$

where

$$
\begin{aligned}
\varsigma_{0}^{2} & :=\varsigma_{0}^{2}\left(p, B, \alpha_{0}\right)=\sigma_{0}^{2}(1+\widetilde{\tau}) \frac{\left(B^{2}-1\right)^{3}}{B^{4} \log ^{2} B}, \\
\sigma_{0}^{2} & :=\sigma_{0}^{2}\left(p, \alpha_{0}\right)=\frac{2}{2^{4 p-\alpha_{0}}} \frac{\Gamma\left(4 p+1-\alpha_{0}\right)}{\left(\Gamma\left(2 p+1-\alpha_{0} / 2\right)\right)^{2}}, \\
\widetilde{\tau}: & =\frac{1}{B^{2}}\left(\left(B^{2}+1\right)\left(\widetilde{\tau}_{0}+\widetilde{\tau}_{2}+\widetilde{\tau}_{0} \widetilde{\tau}_{2}\right)+2 \widetilde{\tau}_{1}-\widetilde{\tau}_{1}^{2}\right)
\end{aligned}
$$

with $\widetilde{\tau}_{0}, \widetilde{\tau}_{1} \widetilde{\tau}_{2}$ as defined in Lemma B.1.

Proof. Again we shall focus on the Taylor expansion

$$
\begin{gathered}
0=\left.\frac{d}{d \alpha} R_{J_{0}, J_{L}}^{M}(\alpha)\right|_{\alpha=\widehat{\alpha}_{J_{0}, J_{L}}^{M}}=\left.S_{J_{0}, J_{L}}^{M}(\alpha)\right|_{\alpha=\alpha_{0}}+\left.Q_{J_{0}, J_{L}}^{M}(\alpha)\right|_{\alpha=\bar{\alpha}}\left(\widehat{\alpha}_{J_{0}, J_{L}}^{M}-\alpha\right), \\
S_{J_{0}, J_{L}}^{M}(\alpha)=\frac{d}{d \alpha} R_{J_{0}, J_{L}}^{M}(\alpha) ; \\
Q_{J_{0}, J_{L}}^{M}(\alpha)=\frac{d^{2}}{d \alpha^{2}} R_{J_{0}, J_{L}}^{M}(\alpha),
\end{gathered}
$$

where $\bar{\alpha} \in\left[\alpha_{0}-\delta_{J_{L}}, \alpha_{0}+\delta_{J_{L}}\right]$, and $\delta_{J} \rightarrow_{p} 0$ as $J_{L} \rightarrow \infty$ by Lemma 4.3. The equation above then leads to

$$
\left(\widehat{\alpha}_{J_{0}, J_{L}}^{M}-\alpha_{0}\right)=-S_{J_{0}, J_{L}}^{M}\left(\alpha_{0}\right)\left(Q_{J_{0}, J_{L}}^{M}(\bar{\alpha})\right)^{-1} .
$$

The proof is readily completed by combining Lemma 4.7 and 4.8 .

Lemma 4.7. Assume Condition 3 holds with $0<4 p+2-\alpha_{0} \leq M$, we have

$$
B^{J_{L}} S_{J_{0}, J_{L}}^{M}\left(\alpha_{0}\right) \rightarrow_{p}-\kappa \frac{I_{p, 0}\left(\alpha_{0}+1\right)}{I_{p, 0}\left(\alpha_{0}\right)} \frac{\log B}{(B+1)} ;
$$

if Condition 4 holds we have

$$
B^{J_{L}} S_{J_{0}, J_{L}}^{M}\left(\alpha_{0}\right) \rightarrow_{D} N\left(0, \sigma_{0}^{2}(1+\widetilde{\tau}) \frac{\log ^{2} B}{B^{2}-1}\right) .
$$


Proof. Note, first of all, that, as in [13], the proof of (4.8) is totally equivalent to the case of (4.7). First of all, we can rewrite $S_{J_{0}, J_{L}}^{M}(\alpha)$ as follows.

$$
\begin{aligned}
S_{J_{0}, J_{L}}^{M}(\alpha) & =\frac{d}{d \alpha} \log \widehat{G}_{J_{0}, J_{L}}^{M}(\alpha)-\frac{d}{d \alpha} \frac{1}{\sum_{j=J_{0}}^{J_{L}} N_{j}} \sum_{j=J_{0}}^{J_{L}} \sum_{k} \log \frac{\beta_{j k ; p}^{2}}{K_{j}^{M}(\alpha)} \\
& =\frac{\widehat{G}_{J_{0}, J_{L}, 1}^{M}(\alpha)}{\widehat{G}_{J_{0}, J_{L}}^{M}(\alpha)}-\frac{1}{\sum_{j=J_{0}}^{J_{L}} N_{j}} \sum_{j=J_{0}}^{J_{L}} \sum_{k} \frac{K_{j, 1}^{M}(\alpha)}{K_{j}^{M}(\alpha)} \\
& =\frac{1}{\sum_{j=J_{0}}^{J_{L}} N_{j}} \sum_{j=J_{0}}^{J_{L}}\left(\frac{K_{j, 1}^{M}(\alpha)}{K_{j}^{M}(\alpha)}\right) \sum_{k}\left(\frac{\beta_{j k ; p}^{2}}{\widehat{G}_{J_{0}, J_{L}}^{M}(\alpha) K_{j}^{M}(\alpha)}-1\right) .
\end{aligned}
$$

We can easily see that

$$
S_{J_{0}, J_{L}}^{M}\left(\alpha_{0}\right)=\frac{G_{0}}{\widehat{G}_{J_{0}, J_{L}}^{M}\left(\alpha_{0}\right)} \bar{S}_{J_{0}, J_{L}}^{M}\left(\alpha_{0}\right)
$$

where

$$
\bar{S}_{J_{0}, J_{L}}^{M}\left(\alpha_{0}\right)=\frac{1}{\sum_{j=J_{0}}^{J_{L}} N_{j}} \sum_{j=J_{0}}^{J_{L}} \frac{K_{j, 1}^{M}\left(\alpha_{0}\right)}{K_{j}^{M}\left(\alpha_{0}\right)} \sum_{k}\left(\frac{\beta_{j k ; p}^{2}}{G_{0} K_{j}^{M}\left(\alpha_{0}\right)}-\frac{\widehat{G}_{J_{0}, J_{L}}^{M}\left(\alpha_{0}\right)}{G_{0}}\right)
$$

and from Lemma 4.2 we have

$$
\frac{G_{0}}{\widehat{G}_{J_{0}, J_{L}, 1}^{M}\left(\alpha_{0}\right)} \rightarrow_{p} 1
$$

Under Condition 3 we have

$$
\begin{gathered}
\lim _{J_{L} \rightarrow \infty} B^{J_{L}} \mathbb{E}\left(\bar{S}_{J_{0}, J_{L}}^{M}\left(\alpha_{0}\right)\right) \\
=\lim _{J_{L} \rightarrow \infty} \frac{B^{J_{L}}}{\sum_{j=J_{0}}^{J_{L}} N_{j}} \sum_{j=J_{0}}^{J_{L}}\left(-\frac{K_{j, 1}^{M}\left(\alpha_{0}\right)}{K_{j}^{M}\left(\alpha_{0}\right)}\right)\left(\frac{\mathbb{E}\left(\widehat{\Lambda}_{j ; p}\right)}{G_{0} K_{j}^{M}\left(\alpha_{0}\right)}-\frac{N_{j}}{\sum_{j=J_{0}}^{J_{L}} N_{j}} \sum_{j=J_{0}}^{J_{L}} \frac{\mathbb{E}\left(\widehat{\Lambda}_{j ; p}\right)}{G_{0} K_{j}^{M}\left(\alpha_{0}\right)}\right) \\
=\lim _{J_{L} \rightarrow \infty} \frac{I_{p, 0}\left(\alpha_{0}+1\right)}{I_{p, 0}\left(\alpha_{0}\right)} \frac{\kappa B^{J_{L}}}{\sum_{j=J_{0}}^{J_{L}} B^{2 j}} \sum_{j=J_{0}}^{J_{L}} \log B^{j} \cdot B^{2 j}\left(B^{-j}-\frac{1}{\sum_{j=J_{0}}^{J_{L}} B^{2 j}} \sum_{j=J_{0}}^{J_{L}} B^{j}\right)+o_{J_{L}} \\
=\lim _{J_{L} \rightarrow \infty}-\kappa \frac{I_{p, 0}\left(\alpha_{0}+1\right)}{I_{p, 0}\left(\alpha_{0}\right)} \frac{\log B}{(B+1)}+o_{J_{L}}(1) ;
\end{gathered}
$$

while under Condition 4 we find immediately

$$
\mathbb{E}\left(\bar{S}_{J_{0}, J_{L}}^{M}\left(\alpha_{0}\right)\right)=o_{J_{L}}(1)
$$

In order to compute the variance of $\bar{S}_{J_{0}, J_{L}}^{M}\left(\alpha_{0}\right)$, we split it into 3 terms (see again [13]):

$$
\operatorname{Var}\left(\bar{S}_{J_{0}, J_{L}}^{M}\left(\alpha_{0}\right)\right)=A+B+C,
$$

where

$$
A=\frac{1}{\left(\sum_{j=J_{0}}^{J_{L}} N_{j}\right)^{2}} \sum_{j_{1}} \sum_{j_{2}}\left(\frac{K_{j_{1}, 1}^{M}\left(\alpha_{0}\right)}{K_{j_{1}}^{M}\left(\alpha_{0}\right)} \frac{K_{j_{2}, 1}^{M}\left(\alpha_{0}\right)}{K_{j_{2}}^{M}\left(\alpha_{0}\right)}\right) \operatorname{Cov}\left(\frac{\sum_{k_{1}} \beta_{j_{1} k_{1} ; p}^{2}}{G_{0} K_{j_{1}}^{M}\left(\alpha_{0}\right)}, \frac{\sum_{k_{2}} \beta_{j_{2} k_{2} ; p}^{2}}{G_{0} K_{j_{2}}^{M}\left(\alpha_{0}\right)}\right)
$$




$$
\begin{gathered}
B=\frac{1}{\left(\sum_{j=J_{0}}^{J_{L}} N_{j}\right)^{2}} \sum_{j_{1}} \sum_{j_{2}}\left(\frac{K_{j_{1}, 1}^{M}\left(\alpha_{0}\right)}{K_{j_{1}}^{M}\left(\alpha_{0}\right)} \frac{K_{j_{2}, 1}^{M}\left(\alpha_{0}\right)}{K_{j_{2}}^{M}\left(\alpha_{0}\right)}\right) N_{j_{1}} N_{j_{2}} \operatorname{Var}\left(\frac{\widehat{G}_{J_{0}, J_{L}}^{M}\left(\alpha_{0}\right)}{G_{0}}\right), \\
C=\frac{-2}{\left(\sum_{j=J_{0}}^{J_{L}} N_{j}\right)^{2}} \sum_{j_{1}} \sum_{j_{2}}\left(\frac{K_{j_{1}, 1}^{M}\left(\alpha_{0}\right)}{K_{j_{1}}^{M}\left(\alpha_{0}\right)} \frac{K_{j_{2}, 1}^{M}\left(\alpha_{0}\right)}{K_{j_{2}}^{M}\left(\alpha_{0}\right)}\right) \operatorname{Cov}\left(\frac{\sum_{k_{1}} \beta_{j_{1} k_{1} ; p}^{2}}{G_{0} K_{j}^{M}\left(\alpha_{0}\right)}, N_{j_{2}} \frac{\widehat{G}_{J_{0}, J_{L}}^{M}\left(\alpha_{0}\right)}{G_{0}}\right) .
\end{gathered}
$$

By fixing $j=j_{1}, \Delta j=j_{2}-j_{1}$, we have:

$$
\begin{aligned}
A= & \frac{1}{\left(\sum_{j=J_{0}}^{J_{L}} N_{j}\right)^{2}} \sum_{j=J_{0}}^{J_{L}} \sum_{\Delta=J_{0}-j}^{J_{L}-j}\left(\frac{K_{j, 1}^{M}\left(\alpha_{0}\right)}{K_{j}^{M}\left(\alpha_{0}\right)} \frac{K_{j+\Delta j, 1}^{M}\left(\alpha_{0}\right)}{K_{j+\Delta j}^{M}\left(\alpha_{0}\right)}\right) \\
& \times \operatorname{Cov}\left(\frac{\sum_{k_{1}} \beta_{j k_{1} ; p}^{2}}{G_{0} K_{j}^{M}\left(\alpha_{0}\right)}, \frac{\sum_{k_{2}} \beta_{j+\Delta j k_{2} ; p}^{2}}{G_{0} K_{j+\Delta j}^{M}\left(\alpha_{0}\right)}\right) \\
= & \frac{\left(B^{2}-1\right) \log ^{2} B}{B^{2} B^{2 J_{L}}} \sigma_{0}^{2}\left(\left(1+\tau_{0}\right) J_{L}^{2}-\left(\frac{2}{B^{2}-1}\left(1+\widetilde{\tau}_{1}\right)\right) J_{L}\right. \\
& \left.+\frac{B^{2}+1}{\left(B^{2}-1\right)^{2}}\left(1+\widetilde{\tau}_{2}\right)\right)+o\left(B^{-2 J_{L}}\right),
\end{aligned}
$$

by applying Lemmas B.1 and A.2. On the other hand, from Lemma 4.1 we obtain

$$
\begin{aligned}
B & =\frac{1}{\left(\sum_{j=J_{0}}^{J_{L}} N_{j}\right)^{2}} \sum_{j_{1}} \sum_{j_{2}}\left(\frac{K_{j_{1}, 1}^{M}\left(\alpha_{0}\right)}{K_{j_{1}}^{M}\left(\alpha_{0}\right)} \frac{K_{j_{2}, 1}^{M}\left(\alpha_{0}\right)}{K_{j_{2}}^{M}\left(\alpha_{0}\right)}\right) N_{j_{1}} N_{j_{2}} \operatorname{Var}\left(\frac{\widehat{G}_{J_{0}, J_{L}}^{M}\left(\alpha_{0}\right)}{G_{0}}\right) \\
& =\frac{\sigma_{0}^{2}}{\left(\sum_{j=J_{0}}^{J_{L}} B^{2 j}\right)^{4}}\left(\sum_{j_{1}=J_{0}}^{J_{L}} \log B^{j_{1}} B^{2 j_{1}}\right)\left(\sum_{j_{2}=J_{0}}^{J_{L}} \log B^{j_{2}} B^{2 j_{2}}\right) \sum_{j_{1}=J_{0}}^{J_{L}} B^{2 j} \sum_{\Delta j=-J_{0}-j}^{J_{L}-j} B^{\alpha_{0} \Delta j} \tau_{B}(\Delta j) \\
& =\frac{\sigma_{0}^{2}}{\left(\sum_{j=J_{0}}^{J_{L}} B^{2 j}\right)^{4}}\left(\sum_{j=J_{0}}^{J_{L}} \log B^{j} B^{2 j}\right)^{2}\left(\frac{B^{2}}{B^{2}-1}\left(1+\widetilde{\tau}_{0}\right) B^{2 J_{L}}\right)+o\left(B^{2 J_{L}}\right) \\
& =\frac{\left(B^{2}-1\right) \log ^{2} B}{B^{2}} \sigma_{0}^{2} B^{-2 J_{L}}\left(\left(J_{L}-\frac{1}{B^{2}-1}\right)^{2}\left(1+\widetilde{\tau}_{0}\right)\right) .
\end{aligned}
$$

Finally, we have that

$$
\begin{aligned}
C= & \frac{-2}{\left(\sum_{j=J_{0}}^{J_{L}} N_{j}\right)^{3}}\left(\sum_{j=J_{0}}^{J_{L}} \log B^{j} \operatorname{Cov}\left(\frac{\sum_{k_{1}} \beta_{j_{1} k_{1} ; p}^{2}}{G_{0} K_{j}^{M}\left(\alpha_{0}\right)}, \sum_{j 3=J_{0}}^{J_{L}} \frac{\sum_{k_{1}} \beta_{j_{3} k_{1} ; p}^{2}}{G_{0} K_{j_{3}}^{M}\left(\alpha_{0}\right)}\right)\right) \\
& \times\left(\sum_{j_{2}=J_{0}}^{J_{L}} B^{2 j_{2}} \log B^{j_{2}}\right)+o\left(B^{2 J_{L}}\right) \\
= & -2 \sigma_{0}^{2} \frac{\left(B^{2}-1\right) \log ^{2} B}{B^{2}} B^{-2 J_{L}}\left(\left(\left(1+\widetilde{\tau}_{0}\right) J_{L}-\left(\frac{1}{B^{2}-1}\left(1+\widetilde{\tau}_{1}\right)\right)\right)\right. \\
& \left.\times\left(J_{L}-\frac{1}{B^{2}-1}\right)\right)+o\left(B^{2 J_{L}}\right) .
\end{aligned}
$$


Summing all these terms, we obtain

$$
A+B+C=\sigma_{0}^{2} \frac{B^{2} \log ^{2} B}{\left(B^{2}-1\right)}(1+\widetilde{\tau}) B^{-2 J_{L}}+o\left(B^{2 J_{L}}\right) .
$$

We can use the Lemma B.1 to observe that

$$
\operatorname{Var}\left(\bar{S}_{J_{0}, J_{L}}^{M}\left(\alpha_{0}\right)\right)=\frac{\sigma_{0}^{2}(1+\widetilde{\tau})}{\left(\sum_{j=J_{0}}^{J_{L}} B^{2 j}\right)^{3}}\left(Z_{J_{L}}+o_{J_{L}}(1)\right) .
$$

Hence we have

$$
\lim _{J_{L} \rightarrow \infty} B^{2 J_{L}} \operatorname{Var}\left(\bar{S}_{J_{0}, J_{L}}^{M}\left(\alpha_{0}\right)\right)=\frac{\sigma_{0}^{2}(1+\widetilde{\tau}) B^{2} \log ^{2} B}{\left(B^{2}-1\right)} .
$$

To prove (4.9), it remains to study the behaviour the fourth order cumulants, observing that this statistics belong to the second order Wiener chaos with respect to a Gaussian white noise random measure (see [42, [13]). Let

$$
B^{J_{L}} S_{J_{L}}\left(\alpha_{0}\right)=\frac{1}{B^{J_{L}}} \sum_{j}\left(A_{j}+B_{j}\right)
$$

where

$$
\begin{aligned}
& A_{j}=B^{2 j} \log B^{j}\left\{\frac{\sum_{k} \beta_{j k}^{2}}{N_{j} G_{0} K_{j}\left(\alpha_{0}\right)}-1\right\}, \\
& B_{j}=B^{2 j} \log B^{j}\left\{\frac{\widehat{G}_{J_{L}}\left(\alpha_{0}\right)}{G_{0}}-1\right\} .
\end{aligned}
$$

In the Appendix, Lemma C.1 proves that:

$$
\begin{aligned}
\frac{1}{B^{4 J_{L}}} \operatorname{cum}\left\{\sum_{l_{1}}\left(A_{j_{1}}+B_{j_{1}}\right)\right. & \left., \sum_{l_{2}}\left(A_{j_{2}}+B_{j_{2}}\right), \sum_{l_{3}}\left(A_{j_{3}}+B_{j_{3}}\right), \sum_{l_{4}}\left(A_{j_{4}}+B_{j_{4}}\right)\right\} \\
= & O_{J_{L}}\left(\frac{J_{L}^{4} \log ^{4} B}{B^{2 J_{L}}}\right) .
\end{aligned}
$$

Exactly as in $\mathbf{1 2}$ and $\mathbf{1 3}$, the Central Limit Theorem follows from results in 42.

Lemma 4.8. Assume Condition 1 holds with $0<4 p+2-\alpha_{0} \leq Q$. Then, for $\bar{\alpha} \in\left[\alpha_{0}-\delta_{J_{L}}, \alpha_{0}+\delta_{J_{L}}\right]$, we have

$$
Q_{J_{0}, J_{L}}^{M}(\bar{\alpha}) \rightarrow_{p} \frac{B^{2} \log ^{2} B}{\left(B^{2}-1\right)^{2}} .
$$

Proof. The procedure is totally analogue to Lemma 19 in $\mathbf{1 3}$. We obtain:

$$
\begin{aligned}
Q_{J_{0}, J_{L}}^{M}(\alpha)= & \frac{G_{J_{0}, J_{L}, 2}^{M}(\alpha) G_{J_{0}, J_{L}}^{M}(\alpha)-\left(G_{J_{0}, J_{L} 1}^{M}(\alpha)\right)^{2}}{\left(G_{J_{0}, J_{L}}^{M}(\alpha)\right)^{2}} \\
& +\frac{1}{\sum_{j} N_{j}} \sum_{j} N_{j} \frac{K_{j, 2}^{M}(\alpha) K_{j}^{M}(\alpha)-\left(K_{j, 1}^{M}(\alpha)\right)^{2}}{\left(K_{j}^{M}(\alpha)\right)^{2}}
\end{aligned}
$$




$$
\begin{gathered}
=\frac{\left(\sum_{j} N_{j} \frac{K_{j}^{M}\left(\alpha_{0}\right)}{K_{j}^{M}(\alpha)}\left(2\left(\frac{K_{j, 1}^{M}(\alpha)}{K_{j}^{M}(\alpha)}\right)^{2}-\frac{K_{j, 2}^{M}(\alpha)}{K_{j}^{M}(\alpha)}\right)\right)\left(\sum_{j} N_{j} \frac{K_{j}^{M}\left(\alpha_{0}\right)}{K_{j}^{M}(\alpha)}\right)}{\left(\sum_{j} N_{j} \frac{K_{j}^{M}\left(\alpha_{0}\right)}{K_{j}^{M I}(\alpha)}\right)^{2}} \\
-\frac{\left(\sum_{j} N_{j} \frac{K_{j}^{M}\left(\alpha_{0}\right)}{K_{j}^{M}(\alpha)}\left(-\frac{K_{j, 1}^{M}(\alpha)}{K_{j}^{M}(\alpha)}\right)\right)^{2}}{\left(\sum_{j} N_{j} \frac{K_{j}^{M}\left(\alpha_{0}\right)}{K_{j}^{M}(\alpha)}\right)^{2}}+\frac{1}{\sum_{j} N_{j}} \sum_{j} N_{j} \frac{K_{j, 2}^{M}(\alpha) K_{j}^{M}(\alpha)-\left(K_{j, 1}^{M}(\alpha)\right)^{2}}{\left(K_{j}^{M}(\alpha)\right)^{2}} .
\end{gathered}
$$

$Q_{J_{0}, J_{L}}^{M}(\alpha)$ can be rewritten as the sum of three terms:

$$
Q_{J_{0}, J_{L}}^{M}(\alpha)=Q_{1}(\alpha)+Q_{2}(\alpha)+Q_{3}(\alpha),
$$

where:

$$
\begin{aligned}
& Q_{1}(\alpha)=\frac{Q_{1}^{\text {num }}(\alpha)}{Q_{1}^{\text {den }}(\alpha)} \\
& =\frac{\left(\sum_{j} N_{j} \frac{K_{j}^{M}\left(\alpha_{0}\right)}{K_{j}^{M}(\alpha)}\left(\frac{K_{j, 1}^{M}(\alpha)}{K_{j}^{M}(\alpha)}\right)^{2}\right)\left(\sum_{j} N_{j} \frac{K_{j}^{M}\left(\alpha_{0}\right)}{K_{j}^{M}(\alpha)}\right)-\left(\sum_{j} N_{j} \frac{K_{j}^{M}\left(\alpha_{0}\right)}{K_{j}^{M}(\alpha)}\left(-\frac{K_{j, 1}^{M}(\alpha)}{K_{j}^{M}(\alpha)}\right)\right)^{2}}{\left(\sum_{j} N_{j} \frac{K_{j}^{M}\left(\alpha_{0}\right)}{K_{j}^{M}(\alpha)}\right)^{2}}, \\
& Q_{2}(\alpha)=\frac{Q_{2}^{\text {num }}(\alpha)}{Q_{2}^{\text {den }}(\alpha)} \\
& =\frac{\left(\sum_{j} N_{j}\right)\left(\sum_{j} N_{j} \frac{K_{j}^{M}\left(\alpha_{0}\right)}{K_{j}^{M}(\alpha)}\left(\frac{K_{j, 1}^{M}(\alpha)}{K_{j}^{M}(\alpha)}\right)^{2}\right)-\left(\sum_{j} N_{j} \frac{K_{j}^{M}\left(\alpha_{0}\right)}{K_{j}^{M}(\alpha)}\right)\left(\sum_{j} N_{j}\left(\frac{K_{j, 1}^{M}(\alpha)}{K_{j}^{M}(\alpha)}\right)^{2}\right)}{\left(\sum_{j} N_{j} \frac{K_{j}^{M}\left(\alpha_{0}\right)}{K_{j}^{M}(\alpha)}\right) \sum_{j} N_{j}}, \\
& Q_{3}(\alpha)=\frac{Q_{3}^{\text {num }}(\alpha)}{Q_{2}^{\text {den }}(\alpha)} \\
& =\frac{\left(\sum_{j} N_{j} \frac{K_{j}^{M}\left(\alpha_{0}\right)}{K_{j}^{M}(\alpha)}\right)\left(\sum_{j} N_{j} \frac{K_{j, 2}^{M}(\alpha)}{K_{j}^{M}(\alpha)}\right)-\left(\sum_{j} N_{j}\right)\left(\sum_{j} N_{j} \frac{K_{j}^{M}\left(\alpha_{0}\right)}{K_{j}^{M}(\alpha)} \frac{K_{j, 2}^{M}(\alpha)}{K_{j}^{M}(\alpha)}\right)}{\left(\sum_{j} N_{j} \frac{K_{j}^{M}\left(\alpha_{0}\right)}{K_{j}^{M}(\alpha)}\right) \sum_{j} N_{j}} .
\end{aligned}
$$

The next step consists in showing that:

$$
Q_{2}(\alpha)+Q_{3}(\alpha)=o_{J_{L}}(1)
$$

Using Corollary $1, Q_{2}^{\text {num }}(\alpha)$ can be written as:

$$
\begin{aligned}
Q_{2}^{\text {num }}(\alpha) & \left(\sum_{j} N_{j}\right)\left(\sum_{j} N_{j} \frac{K_{j}^{M}\left(\alpha_{0}\right)}{K_{j}^{M}(\alpha)}\left(\log ^{2} B^{j}+2 \frac{I_{p, 1}(B)}{I_{p, 0}(B)} \log B^{j}+\left(\frac{I_{p, 1}(B)}{I_{p, 0}(B)}\right)^{2}+o_{J_{L}}(1)\right)\right) \\
& -\left(\sum_{j} N_{j} \frac{K_{j}^{M}\left(\alpha_{0}\right)}{K_{j}^{M}(\alpha)}\right)\left(\sum_{j} N_{j}\left(\log ^{2} B^{j}+2 \frac{I_{p, 1}(B)}{I_{p, 0}(B)} \log B^{j}+\left(\frac{I_{p, 1}(B)}{I_{p, 0}(B)}\right)^{2}+o_{J_{L}}(1)\right)\right),
\end{aligned}
$$

while $Q_{3}^{\text {num }}(\alpha)$ becomes:

$$
Q_{3}^{\text {num }}(\alpha)
$$


HIGH-FREQUENCY TAIL INDEX ESTIMATION BY NEARLY TIGHT FRAMES 21

$$
\begin{aligned}
= & \left(\sum_{j} N_{j} \frac{K_{j}^{M}\left(\alpha_{0}\right)}{K_{j}^{M}(\alpha)}\right)\left(\sum_{j} N_{j}\left(\log B^{2 j}+2 \frac{I_{p, 1}(B)}{I_{p, 0}(B)} \log B^{j}+\frac{I_{p, 2}(B)}{I_{p, 0}(B)}+o_{J_{L}}(1)\right)\right) \\
& -\left(\sum_{j} N_{j}\right)\left(\sum_{j} N_{j} \frac{K_{j}\left(\alpha_{0}\right)}{K_{j}(\alpha)}\left(\log B^{2 j}+2 \frac{I_{p, 1}(B)}{I_{p, 0}(B)} \log B^{j}+\frac{I_{p, 2}(B)}{I_{p, 0}(B)}+o_{J_{L}}(1)\right)\right),
\end{aligned}
$$

so that:

$$
\frac{Q_{2}^{\text {num }}(\alpha)+Q_{3}^{\text {num }}(\alpha)}{Q_{2}^{\text {den }}(\alpha)}=o_{J_{L}}(1)
$$

It remains to study $Q_{2}^{\text {den }}(\alpha)$; by Propositions 1 and 2 , we have:

$$
\begin{aligned}
& \lim _{J_{L} \rightarrow \infty} \frac{1}{B^{2\left(2+\frac{\alpha-a_{0}}{2}\right) J_{L}}}\left(\sum_{j} N_{j} \frac{K_{j}^{M}\left(\alpha_{0}\right)}{K_{j}^{M}(\alpha)}\right)\left(\sum_{j} N_{j}\right) \\
= & \lim _{J_{L} \rightarrow \infty} \frac{c_{B}^{2} I_{p}\left(B, \alpha-\alpha_{0}\right)}{B^{2\left(2+\frac{\alpha-a_{0}}{2}\right) J_{L}}}\left(\sum_{j} B^{2 j\left(1+\frac{\alpha-\alpha_{0}}{2}\right)}\right)\left(\sum_{j} B^{2 j}\right) \\
= & c_{B}^{2} I_{p}\left(B, \alpha-\alpha_{0}\right) \frac{B^{2\left(1+\frac{\alpha-a_{0}}{2}\right)}}{B^{2\left(1+\frac{\alpha-a_{0}}{2}\right)}-1} \frac{B^{2}}{B^{2}-1}>0 .
\end{aligned}
$$

Finally, we prove that $Q_{1}\left(\bar{\alpha}_{L}\right) \rightarrow_{p} \frac{B^{2} \log ^{2} B}{\left(B^{2}-1\right)^{2}}$. Using again Proposition 1 and Corollary 1, we write the numerator $Q_{1}^{\text {num }}(\alpha)$ as:

$$
\begin{gathered}
Q_{1}^{\text {num }}(\alpha) \\
=\left(\sum_{j} \frac{K_{j}^{M}\left(\alpha_{0}\right)}{K_{j}^{M}(\alpha)} N_{j}\right)\left(\sum_{j} N_{j} \frac{K_{j}^{M}\left(\alpha_{0}\right)}{K_{j}^{M}(\alpha)}\left(\log B^{j}+\frac{I_{p, 1}(B)}{I_{p, 0}(B)}\right)^{2}\right) \\
-\left(\sum_{j} N_{j} \frac{K_{j}^{M}\left(\alpha_{0}\right)}{K_{j}^{M}(\alpha)}\left(\log B^{j}+\frac{I_{p, 1}(B)}{I_{p, 0}(B)}\right)\right)^{2} \\
=\left(\sum_{j} \frac{K_{j}^{M}\left(\alpha_{0}\right)}{K_{j}^{M}(\alpha)} N_{j}\left(\sum_{j} N_{j} \frac{K_{j}^{M}\left(\alpha_{0}\right)}{K_{j}^{M}(\alpha)} \log ^{2} B^{j}\right)\right)-\left(\sum_{j} N_{j} \frac{K_{j}^{M}\left(\alpha_{0}\right)}{K_{j}^{M}(\alpha)} \log B^{j}\right)^{2}
\end{gathered}
$$

Let $s=2\left(1+\frac{\alpha-a_{0}}{2}\right)$; by applying Corollary 2 we have:

$$
\begin{aligned}
\lim _{J_{L} \rightarrow \infty} \frac{1}{B^{2 s J_{L}}} Q_{1}^{\text {num }}(\alpha) & =\lim _{J_{L} \rightarrow \infty} \frac{c_{B}^{2} I_{p}\left(B, \alpha-\alpha_{0}\right)}{B^{2 s J_{L}}} Z_{J_{L}}(s) \\
& =\log ^{2} B \frac{B^{3 s}}{\left(B^{s}-1\right)^{4}} c_{B}^{2} I\left(B, \alpha_{0}, \alpha\right)
\end{aligned}
$$


It remains to study $Q_{1}^{d e n}(\alpha)$; by using again (A.6) and Proposition 2

$$
\begin{aligned}
\lim _{J_{L} \rightarrow \infty} \frac{1}{B^{2 s J_{L}}} Q_{1}^{d e n}(\alpha) & =\lim _{J_{L} \rightarrow \infty} \frac{c_{B}^{2} I_{p}\left(B, \alpha-\alpha_{0}\right)}{B^{2 s J_{L}}}\left(\sum_{j} B^{s j}\right)^{2} \\
& =c_{B}^{2} I_{p}\left(B, \alpha-\alpha_{0}\right)\left(\frac{B^{s}}{B^{s}-1}\right)^{2}
\end{aligned}
$$

Hence

$$
\lim _{J_{L} \rightarrow \infty} Q_{J_{0}, J_{L}}^{M}(\alpha)=\frac{B^{2\left(1+\frac{\alpha-a_{0}}{2}\right)} \log ^{2} B}{\left(B^{2\left(1+\frac{\alpha-a_{0}}{2}\right)}-1\right)^{2}}
$$

For the consistency of $\widehat{\alpha}_{L}$, for $\bar{\alpha}_{L} \in\left[\alpha_{0}-\widehat{\alpha}_{L}, \alpha_{0}+\widehat{\alpha}_{L}\right]$, we have

$$
Q_{J_{0}, J_{L}}^{M}\left(\bar{\alpha}_{L}\right) \longrightarrow_{p} \frac{B^{2} \log ^{2} B}{\left(B^{2}-1\right)^{2}} .
$$

\section{Narrow band estimates}

From Theorem 4.6, it is evident that, under Condition 3, the presence of the bias term does not allow asymptotic inference. As in $\mathbf{1 2}$ and $\mathbf{1 3}$, we suggest a narrow-band strategy, developed only on the higher tail of the power spectrum, which allows us to avoid the problem due to the nuisance parameter. We start from the following

Definition 5.1. The Narrow-Band Mexican Needlet Whittle estimator for the parameters $\vartheta=(\alpha, G)$ is provided by

$$
\left(\widehat{\alpha}_{J_{1} ; J_{L}}^{M}, \widehat{G}_{J_{1} ; J_{L}}^{M}\right):=\arg \min _{\alpha, G} \sum_{j=J_{1}}^{J_{L}}\left[\frac{\sum_{k} \beta_{j k ; p}^{2}}{G K_{j}^{M}(\alpha)}-\sum_{k=1}^{N_{j}} \log \left(\frac{\beta_{j k ; p}^{2}}{G K_{j}^{M}(\alpha)}\right)\right],
$$

or equivalently:

$$
\begin{aligned}
\widehat{\alpha}_{J_{1}, J_{L}}^{M} & =\arg \min _{\alpha} R_{J_{1} ; J_{L}}^{M}\left(\alpha, \widehat{G}_{J_{1} ; J_{L}}^{M}(\alpha)\right), \\
R_{J_{1} ; J_{L}}^{M}(\alpha) & =\left(\log \widehat{G}_{J_{1} ; J_{L}}^{M}(\alpha)+\frac{1}{\sum_{j=J_{0}}^{J_{L}} N_{j}} \sum_{J_{1}=J_{1}}^{J_{L}} N_{j} \log K_{j}^{M}(\alpha)\right),
\end{aligned}
$$

where $0<J_{1}<J_{L}$ is chosen such that $B^{J_{L}}-B^{J_{1}} \rightarrow \infty$ and

$$
B^{J_{1}}=B^{J_{L}}\left(1-g\left(J_{L}\right)\right), J_{1}=J_{L}+\frac{\log \left(1-g\left(J_{L}\right)\right)}{\log B} .
$$

We choose $0<g\left(J_{L}\right)<1$ s.t. $\lim _{J_{L} \rightarrow \infty} g\left(J_{L}\right)=0$ and $\lim _{J_{L} \rightarrow \infty} J_{L} g\left(J_{L}\right)^{\frac{3}{2}}=0$.

For notational simplicity $B^{J_{1}}$ is defined as an integer (if this isn't the case, modified arguments taking integer parts are completely trivial). For definiteness, we can take for instance $g\left(J_{L}\right)=J_{L}^{-3}$.

Theorem 5.2. Let $\widehat{\alpha}_{J_{L} ; J_{1}}$ defined as in (5.1). Then under Condition 3 we have 


$$
g\left(J_{L}\right)^{\frac{1}{2}} B^{J_{L}}\left(\widehat{\alpha}_{J_{1} ; J_{L}}-\alpha_{0}\right) \stackrel{d}{\longrightarrow} \mathcal{N}\left(0, \frac{\sigma_{0}^{2}(1+\widetilde{\tau})}{\Phi(B)}\right)
$$

where

$$
\Phi(B):=\log ^{2} B \frac{B^{2}}{\left(B^{2}-1\right)^{2}}\left(\frac{4}{\left(B^{2}-1\right)}+2\left(\frac{\log B-1}{\log B}\right)\right) .
$$

PROOF. The proof is very similar to the full band case, hence we provide here just the main differences. Consider:

$$
\begin{aligned}
S_{J_{1} ; J_{L}}(\alpha) & =\frac{d}{d \alpha} R_{J_{1} ; J_{L}}^{M}(\alpha) ; \\
Q_{J_{1} ; J_{L}}^{M}(\alpha) & =\frac{d^{2}}{d \alpha^{2}} R_{J_{1} ; J_{L}}^{M}(\alpha)
\end{aligned}
$$

Let

$$
\bar{S}_{J_{1} ; J_{L}}^{M}\left(\alpha_{0}\right)=\frac{-1}{\sum_{j=J_{1}}^{J_{L}} N_{j}} \sum_{j=J_{1}}^{J_{L}} \frac{K_{j, 1}^{M}\left(\alpha_{0}\right)}{K_{j}^{M}\left(\alpha_{0}\right)} \sum_{k=1}^{N_{j}}\left(\frac{\beta_{j k ; p}^{2}}{G\left(\alpha_{0}\right) K_{j}^{M}\left(\alpha_{0}\right)}-\frac{\widehat{G}_{J_{1} ; J_{L}}^{M}\left(\alpha_{0}\right)}{G_{0}}\right) .
$$

Simple calculations based on Proposition 2 lead to

$$
\begin{aligned}
\left(\sum_{j=J_{1}}^{J_{L}} B^{2 j}\right)^{n} & =\frac{B^{2 n}}{\left(B^{2}-1\right)^{n}}\left(B^{2 J_{L}}-B^{2\left(J_{1}-1\right)}\right)^{n}+o\left(B^{2 n J_{L}}\right) \\
& =B^{n J_{L}}+O\left(B^{2 n J_{L}} g\left(J_{L}\right)\right)
\end{aligned}
$$

We have:

$$
\begin{gathered}
\lim _{J_{L} \rightarrow \infty} \frac{B^{J_{L}}}{J_{L} g\left(J_{L}\right)} \mathbb{E}\left(\bar{S}_{J_{0}, J_{L}}^{M}\left(\alpha_{0}\right)\right) \\
=\lim _{J_{L} \rightarrow \infty} \frac{B^{J_{L}}}{J_{L} g\left(J_{L}\right)} \kappa \frac{I_{p, 0}\left(\alpha_{0}+1\right)}{I_{p, 0}\left(\alpha_{0}\right)} \\
\times\left(\sum_{j=J_{1}}^{J_{L}} \log B^{j} \cdot B^{2 j}\left(B^{-j}-B^{-J_{L}}\left(\frac{B-1}{B}+\frac{g\left(J_{L}\right)}{B}\right)\right)\right)+o_{J_{L}} \\
=\lim _{J_{L} \rightarrow \infty}-\kappa \frac{I_{p, 0}\left(\alpha_{0}+1\right)}{I_{p, 0}\left(\alpha_{0}\right)} \frac{\log B}{B+1}+o_{J_{L}}(1)
\end{gathered}
$$

As in full band case, we collect out all the covariance terms defined in Lemma B.1 and following Corollary 2, we have

$$
\operatorname{Var}\left(\bar{S}_{J_{L} ; J_{1}}\left(\alpha_{0}\right)\right)=\frac{\sigma_{0}^{2}(1+\widetilde{\tau})}{\left(\sum_{j=J_{1}}^{J_{L}} B^{2 j}\right)^{3}}\left(Z_{J_{1} ; J_{L}}(2)+o_{J_{L}}(1)\right) .
$$

After some manipulations, we have:

$$
\frac{1}{B^{4 J_{L}}} Z_{J_{1} ; J_{L}}
$$




$$
\begin{aligned}
& =\left(1-\frac{\left(1-g\left(J_{L}\right)\right)^{2}}{B^{2}}\right)^{2}-\frac{\left(B^{2}-1\right)^{2}}{B^{4}}\left(1-g\left(J_{L}\right)\right)^{2}\left(1-\log _{B}\left(1-g\left(J_{L}\right)\right)\right)^{2} \\
& =\left(\frac{B^{2}-1}{B^{2}}\right)^{2} \Delta Z_{J_{L} ; J_{1}}\left(g\left(J_{L}\right)\right)+O\left(g\left(J_{L}\right)^{2}\right) \\
(5.3) & =\left(\frac{B^{2}-1}{B^{2}}\right)^{2}\left(\frac{4}{\left(B^{2}-1\right)}+\left(2-\frac{2}{\log B}\right)\right) g\left(J_{L}\right)+O\left(B^{4 J_{L}} g\left(J_{L}\right)^{2}\right),
\end{aligned}
$$

where

$$
\begin{aligned}
\Delta Z_{J_{1} ; J_{L}}\left(g\left(J_{L}\right)\right) & =\left(1+\frac{4 g\left(J_{L}\right)}{\left(B^{2}-1\right)}\right)-\left(1-2 g\left(J_{L}\right)\right)\left(1+\frac{1}{\log B} g\left(J_{L}\right)\right)^{2} \\
& =\left(\frac{4}{\left(B^{2}-1\right)}+\left(2-\frac{2}{\log B}\right)\right) g\left(J_{L}\right)
\end{aligned}
$$

Thus we have

$$
Z_{J_{L} ; J_{1}}(2)=B^{4 J_{L}} \Phi(B) g\left(J_{L}\right)+O\left(B^{4 J_{L}} g\left(J_{L}\right)^{2}\right) .
$$

Note that $\Phi(B)>0$ for $B>1$.

Hence we have

$$
\operatorname{Var}\left(\bar{S}_{J_{1} ; J_{L}}^{M}\left(\alpha_{0}\right)\right)=\sigma_{0}^{2}(1+\widetilde{\tau}) \Phi(B) g\left(J_{L}\right) B^{-2 J_{L}},
$$

Consider now $Q_{J_{L} ; J_{1}}(\alpha)$, which we rewrite as

$$
Q_{J_{1} ; J_{L}}^{M}(\alpha)=\frac{Q_{n u m}(\alpha)}{Q_{\text {den }}(\alpha)} .
$$

Following a procedure similar to Lemma 4.8 we have

$$
Q_{\text {num }}(\alpha)=c_{B}^{2} G_{0}^{2} I_{p}\left(B, \alpha-\alpha_{0}\right) Z_{J_{1} ; J_{L}}(s),
$$

where $s=2\left(1+\frac{\alpha-\alpha_{0}}{2}\right)$. Following (5.3), we obtain

$$
Q_{n u m}(\alpha)=c_{B}^{2} G_{0}^{2} I\left(B, \alpha-\alpha_{0}\right) \Phi(B, s) B^{2 s J_{L}} g\left(J_{L}\right)+O\left(B^{2 s J_{L}} g\left(J_{L}\right)^{2}\right),
$$

where

$$
\Phi(B, s)=\log ^{2} B \frac{B^{s}}{\left(B^{s}-1\right)^{2}}\left(\frac{2 s g\left(J_{L}\right)}{B^{s}-1}+\frac{s \log B-2}{\log B}\right) .
$$

Finally, we obtain

$$
\begin{aligned}
Q_{d e n}(\alpha) & =c_{B}^{2} G_{0}^{2} I\left(B, \alpha-\alpha_{0}\right)\left(\sum_{j=J_{1}}^{J_{L}} B^{s j}\right)^{2} \\
& =c_{B}^{2} G_{0}^{2} I_{p}\left(B, \alpha-\alpha_{0}\right) B^{2 s J_{L}}+o\left(B^{2 s J_{L}}\right) .
\end{aligned}
$$

Hence

$$
Q_{J_{1} ; J_{L}}^{M}(\alpha)=\Phi(B, s) g\left(J_{L}\right)+O\left(B^{2 s J_{L}} g\left(J_{L}\right)^{2}\right),
$$

and, for the consistency of $\alpha$, we have

$$
Q_{J_{1} ; J_{L}}^{M}(\bar{\alpha}) \rightarrow_{p} \Phi(B) g\left(J_{L}\right) .
$$

Thus

$$
\left(\frac{\sigma_{0}^{2}(1+\widetilde{\tau})}{\Phi(B)}\right)^{-\frac{1}{2}} g\left(J_{L}\right)^{\frac{1}{2}} B^{J_{L}} \frac{\bar{S}_{J_{1} ; J_{L}}^{M}\left(\alpha_{0}\right)}{Q_{J_{1} ; J_{L}}^{M}(\bar{\alpha})} \stackrel{d}{\rightarrow} \mathcal{N}(0,1)
$$


as claimed. Finally we can see that

$$
g\left(J_{L}\right)^{\frac{1}{2}} B^{J_{L}} \mathbb{E} \frac{\bar{S}_{J_{1} ; J_{L}}^{M}\left(\alpha_{0}\right)}{Q_{J_{1} ; J_{L}}^{M}(\bar{\alpha})}=O\left(J_{L} \cdot g\left(J_{L}\right)^{\frac{3}{2}}\right) \underset{J_{L \rightarrow \infty}}{\rightarrow} 0 .
$$

\section{The Plug-in procedure}

In this Section, we will present a plug-in estimation procedure for the spectral parameter $\alpha_{0}$ based on the interaction of the approach described here and the one based upon standard needlets introduced in [13. As already mentioned in the Introduction, there already exist in literature Whittle-like estimators for spectral parameter based on spherical harmonics and standard needlets. The former, although characterized by a higher efficiency, can be affected by the presence of masked regions over the sphere, common set-up in Cosmological investigations, because of the lack of localization in the spatial domain. The latter, as one here developed, is not altered by partially observed regions, paying the price of a lower precision. Therefore, our aim is to show that, if $4 p-\alpha_{0}>0$, the spectral parameter

estimator $\widehat{\alpha}_{J_{0}, J_{L}}^{M}$ is more efficient with respect to the standard needlet estimator $\widehat{\alpha}_{J_{L}}$. First of all, observe that

$$
\begin{aligned}
\lim _{J_{L} \rightarrow \infty} B^{2 J_{L}} \operatorname{Var}\left(\widehat{\alpha}_{J_{0}, J_{L}}^{M}-\alpha_{0}\right) & =\sigma_{0}^{2}(1+\widetilde{\tau}) \frac{\left(B^{2}-1\right)^{3}}{B^{4} \log ^{2} B}, \\
\lim _{J_{L} \rightarrow \infty} B^{2 J_{L}} \operatorname{Var}\left(\widehat{\alpha}_{J_{L}}-\alpha_{0}\right) & =\rho_{0}^{2} \frac{\left(B^{2}-1\right)^{3}}{B^{4} \log ^{2} B}
\end{aligned}
$$

see again [13. We can therefore observe that for $4 p-\alpha_{0}>0$,

$$
\sigma_{1}^{2}<\rho_{0}^{2},
$$

where $\sigma_{1}^{2}:=\sigma_{0}^{2}(1+\widetilde{\tau})$. Consider that, for any fixed $p: 4 p>\alpha_{0}, \sigma_{0}^{2}$, which does not depend on $B$, becomes, by the Stirling's formula,

$$
\sigma_{0}^{2} \simeq \frac{2}{\sqrt{\left(\pi\left(2 p-\frac{\alpha_{0}}{2}\right)\right)}} .
$$

We have that $\sigma_{0}^{2}$ is smaller than 1 for $4 p \gtrsim \alpha_{0}-2$, while easy calculations show that $\widetilde{\tau}<1$. On the other hand, as described in [13], $\rho_{0}^{2}=\rho_{0}^{2}\left(\alpha_{0}, B\right)$ is decreasing on $B$ (see also Table 1 ): any attempt to reduce its value will produce an increase of the variance due to the term $\left(B^{2}-1\right)^{3} / B^{4} \log ^{2} B$.

\begin{tabular}{|c|c|c|c|c|c|c|}
\cline { 2 - 7 } \multicolumn{1}{c|}{} & \multicolumn{3}{c|}{ Standard Needlet- $\rho_{0}^{2}$} & \multicolumn{3}{c|}{ Mexican Needlets - $\sigma_{1}^{2}$} \\
\cline { 2 - 7 } \multicolumn{1}{c|}{} & $B=\sqrt[4]{2}$ & $B=\sqrt{ } 2$ & $B=2$ & $p=2$ & $p=3$ & $p=4$ \\
\hline$\alpha_{0}=2$ & 5.00 & 2.24 & 1.16 & 0.62 & 0.49 & 0.42 \\
$\alpha_{0}=3$ & 5.04 & 2.53 & 1.34 & 0.67 & 0.51 & 0.43 \\
$\alpha_{0}=4$ & 5.10 & 2.64 & 1.57 & 0.75 & 0.55 & 0.45 \\
\hline
\end{tabular}

Table 1: Comparison among different values of the variances $\rho_{0}^{2}$ and $\sigma_{0}^{2}$.

Hence, the plug-in procedure can be implemented in two steps:

- First step: compute $\widehat{\alpha}_{J_{L}}$ in the standard needlet framework.

- Second step: if $p>\widehat{\alpha}_{J_{L}} / 4$, compute $\widehat{\alpha}_{J_{0}, J_{L}}^{M}$ by the mexican needlet approach; otherwhise, accept $\widehat{\alpha}_{J_{L}}$. 


\section{Appendix A. Auxiliary results: preliminaries}

The results collected in this section, provided by standard analytical calculations, are here reported to explicit the structure and the behaviour of the function $f_{p}(\cdot)$ defined in in (2.2).

Lemma A.1. Let

$$
W_{2 a, b, s}=\int_{0}^{\infty} t^{2 a} \exp \left(-b t^{2}\right) \log ^{s}(t) d t .
$$

We have

$$
\begin{gathered}
W_{2 a, b, 0}=\frac{b^{-\left(a+\frac{1}{2}\right)}}{2} \Gamma\left(a+\frac{1}{2}\right), \\
W_{2 a, b, 1}=\frac{b^{-\left(a+\frac{1}{2}\right)}}{4}\left[\frac{d}{d a}-\log b\right] \Gamma\left(a+\frac{1}{2}\right)
\end{gathered}
$$

and

$$
W_{2 a, b, 2}=\frac{b^{-\left(a+\frac{1}{2}\right)}}{8}\left[\frac{d^{2}}{d a^{2}}-2 \log b \frac{d}{d a}+\log ^{2} b\right] \Gamma\left(a+\frac{1}{2}\right) .
$$

Proof. Standard calculations lead to

$$
\begin{aligned}
W_{2 a, b, 0} & =\int_{0}^{\infty} t^{2 a} \exp \left(-b t^{2}\right) d t \\
& =\frac{b^{-\left(a+\frac{1}{2}\right)}}{2} \int_{0}^{\infty}\left(b t^{2}\right)^{a-\frac{1}{2}} \exp \left(-b t^{2}\right)(2 b t d t) \\
& =\frac{b^{-\left(a+\frac{1}{2}\right)}}{2} \Gamma\left(a+\frac{1}{2}\right) ;
\end{aligned}
$$

Similarly

$$
\begin{aligned}
W_{2 a, b, 1} & =\int_{0}^{\infty} t^{2 a} \exp \left(-b t^{2}\right) \log t d t \\
& =\frac{1}{2} \int_{0}^{\infty} t^{2 a} \exp \left(-b t^{2}\right) \log \left(b t^{2}\right) d t-\frac{\log b}{2} \int_{0}^{\infty} t^{2 a} \exp \left(-b t^{2}\right) d t \\
& =\frac{b^{-\left(a+\frac{1}{2}\right)}}{4} \int_{0}^{\infty} x^{a-\frac{1}{2}} \exp (-x)[\log x-\log b] d x \\
& =\frac{b^{-\left(a+\frac{1}{2}\right)}}{4}\left[\frac{d}{d a}-\log b\right] \Gamma\left(a+\frac{1}{2}\right) ; \\
W_{a, b, 2}= & \int_{0}^{\infty} t^{2 a} \exp \left(-b t^{2}\right)(\log t)^{2} d t \\
= & \frac{b^{-\left(a+\frac{1}{2}\right)}}{8} \int_{0}^{\infty}\left(b t^{2}\right)^{a-\frac{1}{2}} \exp \left(-b t^{2}\right)\left[\left(\log b t^{2}\right)^{2}-2 \log b \log b t^{2}+\log ^{2} b\right] 2 b t d t \\
= & \frac{b^{-\left(a+\frac{1}{2}\right)}}{8}\left[\frac{d^{2}}{d a^{2}}-2 \log b \frac{d}{d a}+\log ^{2} b\right] \Gamma\left(a+\frac{1}{2}\right) \cdot
\end{aligned}
$$


Lemma A.2. Let $f_{p}(\cdot)$ be defined as in (2.2). Then we have

$$
\sum_{l \geq 1} f_{p}^{a}\left(\frac{l}{B^{j}}\right) l^{n}=\frac{B^{(n+1) j}}{2 a^{a p+\frac{n+1}{2}}} \Gamma\left(a p+\frac{n+1}{2}\right)+o\left(B^{j(n+1)}\right)
$$

Moreover, for $\Delta j \in \mathbb{Z}$, we have

$$
\begin{gathered}
\sum_{l \geq 1} f_{p}^{a_{1}}\left(\frac{l}{B^{j}}\right) f_{p}^{a_{2}}\left(\frac{l}{B^{j+\Delta j}}\right) l^{n} . \\
=\frac{B^{(n+1) j} \tau_{p, a_{1}, a_{2}}(\Delta j)}{2\left(a_{1}+a_{2}\right)^{\left(a_{1}+a_{2}\right) p+\frac{n+1}{2}}} \Gamma\left(\left(a_{1}+a_{2}\right) p+\frac{n+1}{2}\right)+o\left(B^{(n+1) j}\right),
\end{gathered}
$$

where

$$
\tau_{p, a_{1}, a_{2}}(\Delta j)=\left(\frac{a_{1} B^{\Delta j}+a_{2} B^{-\Delta j}}{a_{1}+a_{2}}\right)^{-\left(\left(a_{1}+a_{2}\right) p+\frac{n+1}{2}\right)} B^{\Delta j\left(\left(a_{1}-a_{2}\right) p+\frac{n+1}{2}\right)} .
$$

Proof. Observe that

$$
\begin{aligned}
& \sum_{l \geq 1} f_{p}^{a_{1}}\left(\frac{l}{B^{j}}\right) f_{p}^{a_{2}}\left(\frac{l}{B^{j+\Delta j}}\right) l^{n} \\
& =\sum_{l \geq 1} \exp \left(-l^{2}\left(\frac{a_{1}}{B^{2 j}}+\frac{a_{2}}{B^{2 j+2 \Delta j}}\right)\right)\left(\left(\frac{l}{B^{j}}\right)^{2}\right)^{a_{1} p}\left(\left(\frac{l}{B^{j+\Delta j}}\right)^{2}\right)^{a_{2} p} l^{n} \\
& =\frac{B^{j n}}{B^{2 a_{2} p \Delta j}} \sum_{l \geq 1} \exp \left(-\left(\frac{l}{B^{j}}\right)^{2}\left(\frac{a_{1} B^{2 \Delta j}+a_{2}}{B^{2 \Delta j}}\right)\right)\left(\left(\frac{l}{B^{j}}\right)^{2}\right)^{\left(a_{1}+a_{2}\right) p+\frac{n}{2}} \\
& =\frac{B^{j(n+1)}}{2 B^{2 a_{2} p \Delta j}}\left(\frac{B^{2 \Delta j}}{a_{1} B^{2 \Delta j}+a_{2}}\right)^{\left(a_{1}+a_{2}\right) p+\frac{n+1}{2}} \int_{0}^{+\infty} x^{\left[\left(a_{1}+a_{2}\right) p+\frac{n-1}{2}\right]} \exp (-x) d x+o\left(B^{(n+1) j}\right) \\
& =\frac{B^{j(n+1)}}{2} \frac{B^{2 \Delta j\left(a_{1} p+\frac{n+1}{2}\right)}}{\left(a_{1} B^{2 \Delta j}+a_{2}\right)^{\left(a_{1}+a_{2}\right) p+\frac{n+1}{2}}} \Gamma\left(\left(a_{1}+a_{2}\right) p+\frac{n+1}{2}\right)+o\left(B^{(n+1) j}\right) \\
& =\frac{B^{(n+1) j}}{2\left(a_{1}+a_{2}\right)^{\left(a_{1}+a_{2}\right) p+\frac{n+1}{2}}} \tau_{p, a_{1}, a_{2}}(\Delta j) \Gamma\left(\left(a_{1}+a_{2}\right) p+\frac{n+1}{2}\right)+o\left(B^{(n+1) j}\right) \text {. }
\end{aligned}
$$

Fixing $\Delta j=0, a_{1}=a_{2}=a / 2$, we obtain

$$
\sum_{l \geq 1} f_{p}^{a}\left(\frac{l}{B^{j}}\right) l^{n}=\frac{B^{(n+1) j}}{2 a^{a p+\frac{n+1}{2}}} \Gamma\left(a p+\frac{n+1}{2}\right)+o\left(B^{j(n+1)}\right)
$$

as claimed.

We now investigate the behaviour of the function $K_{j}^{M}(\alpha)$ and its derivatives.

Proposition 1. Let

$$
I_{p, s}(\alpha)=\frac{2}{C_{B}} W_{4 p+1-\alpha, 2, s}=\frac{2}{C_{B}} \int_{0}^{\infty} t^{4 p+1-\alpha} e^{-2 t^{2}}(\log t)^{s} d t, s=0,1,2 .
$$


Then we have

$$
\begin{gathered}
K_{j}^{M}(\alpha)=\left(I_{p, 0}(\alpha)+o_{j}(1)\right) B^{-\alpha j} \\
=\frac{2^{-\left(2 p-\frac{\alpha}{2}+1\right)}}{C_{B}} \Gamma\left(2 p+1-\frac{\alpha}{2}\right) B^{-\alpha j} \\
K_{j, 1}^{M}(\alpha) \quad: \quad=\frac{d}{d \alpha} K_{j}^{M}(\alpha) \\
=\quad-\left(j \log B+\frac{I_{p, 1}(\alpha)}{I_{p, 0}(\alpha)}+o(1)\right) K_{j}^{M}(\alpha) \\
K_{j, 2}^{M}(\alpha):=\frac{d^{2}}{d \alpha^{2}} K_{j}^{M}(\alpha) \\
=\left(j^{2} \log ^{2} B+2 j \log B \frac{I_{p, 1}(\alpha)}{I_{p, 0}(\alpha)}+\frac{I_{p, 2}(\alpha)}{I_{p, 0}(\alpha)}+o(1)\right) K_{j}^{M}(\alpha) .
\end{gathered}
$$

Proof. These proofs follow the ones concerning the scalar needlet case (see 13). We have indeed

$$
\begin{aligned}
K_{j}^{M}(\alpha)= & \frac{1}{C_{B} B^{2 j}} \sum_{l \geq 1}\left(\frac{l}{B^{j}}\right)^{4 p} \exp \left(-2\left(\frac{l}{B^{j}}\right)^{2}\right)(2 l+1) l^{-\alpha} \\
= & B^{(2-\alpha) j} \frac{2}{C_{B} B^{2 j}} \sum_{l \geq 1}\left(\frac{l}{B^{j}}\right)^{4 p} \exp \left(-2\left(\frac{l}{B^{j}}\right)^{2}\right) \frac{\left(l^{1-\alpha}+o\left(l^{1-\alpha}\right)\right)}{B^{(1-\alpha) j}} \\
= & B^{-\alpha j} \frac{2}{C_{B}} W_{4 p+1-\alpha, 2,0}+o_{j}\left(B^{-\alpha j}\right) \\
= & B^{-\alpha j} I_{p, 0}(\alpha)+o_{j}\left(B^{-\alpha j}\right), \\
K_{j, 1}^{M}(\alpha)= & \frac{1}{C_{B} B^{2 j}} \sum_{l \geq 1}\left(\frac{l}{B^{j}}\right)^{4 p} \exp \left(-2\left(\frac{l}{B^{j}}\right)^{2}\right)(2 l+1) l^{-\alpha}(-\log l) \\
= & -K_{j}^{M}(\alpha) \log B^{j}-\frac{2}{C_{B}} B^{-\alpha j}\left(\int t^{4 p+1-\alpha} e^{-2 t^{2}} \log t d t+o_{j}(1)\right) \\
= & -\left(j \log B+\frac{I_{p, 1}(\alpha)}{I_{p, 0}(\alpha)}+o_{j}(1)\right) K_{j}^{M}(\alpha), \\
K_{j, 2}^{M}(\alpha)= & \frac{1}{C_{B} B^{2 j}} \sum_{l \geq 1}\left(\frac{l}{B^{j}}\right)^{4 p} \exp \left(-2\left(\frac{l}{B^{j}}\right)^{2}\right)(2 l+1) l^{-\alpha} \log { }^{2} l \\
= & K_{j}^{M}(\alpha)\left(\log B^{j}\right)^{2}+2 B^{-\alpha j} \log ^{j}\left(\frac{2}{C_{B}} \int t^{4 p+1-\alpha} e^{-2 t^{2}} \log t d t+o(1)\right) \\
& +B^{-\alpha j}\left(\frac{2}{C_{B}} \int t^{4 p+1-\alpha} e^{-2 t^{2}} \log ^{2} t d t+o(1)\right) \\
= & \left(j^{2} \log ^{2} B+2 j \log B \frac{I_{p, 1}(\alpha)}{I_{p, 0}(\alpha)}+\frac{I_{p, 2}(\alpha)}{I_{p, 0}(\alpha)}+o(1)\right) K_{j}^{M}(\alpha) .
\end{aligned}
$$


Corollary 1. From Proposition 1, we have that:

$$
\frac{K_{j}^{M}(\alpha)}{K_{j}^{M}\left(\alpha_{0}\right)}=I_{p}\left(B, \alpha-\alpha_{0}\right) B^{\left(\alpha_{0}-\alpha\right) j}+o\left(B^{\left(\alpha_{0}-\alpha\right) j}\right),
$$

where

$$
I_{p}\left(B, \alpha-\alpha_{0}\right):=(2(2 p+1))^{\frac{\alpha_{0}-\alpha}{2}} .
$$

Proof. The computation above shows that

$$
\begin{aligned}
I_{p, 0}(\alpha) & =\frac{2}{C_{B}} W_{4 p+1-\alpha, 2,0} \\
& =\frac{2^{-\left(2 p-\frac{\alpha}{2}+1\right)}}{C_{B}} \Gamma\left(2 p+1-\frac{\alpha}{2}\right),
\end{aligned}
$$

and following (A.3)

$$
\begin{aligned}
\frac{K_{j}^{M}(\alpha)}{K_{j}^{M}\left(\alpha_{0}\right)} & =B^{\left(\alpha_{0}-\alpha\right) j} 2^{\frac{\alpha_{0}-\alpha}{2}} \frac{\Gamma\left(2 p+1-\frac{\alpha}{2}\right)}{\Gamma\left(2 p+1-\frac{\alpha_{0}}{2}\right)}+o\left(B^{\left(\alpha_{0}-\alpha\right) j}\right) \\
& =B^{\left(\alpha_{0}-\alpha\right) j}(2(2 p+1))^{\frac{\alpha_{0}-\alpha}{2}}+o\left(B^{\left(\alpha_{0}-\alpha\right) j}\right)
\end{aligned}
$$

as claimed.

The next results follow strictly Proposition 27 in $\mathbf{1 3}$, hence we will report the statements, while we will omit the proofs.

Proposition 2. Let $s>0, B>1$. Then

$$
\begin{aligned}
& \sum_{j=J_{0}}^{J_{L}} B^{s j}= \frac{B^{s}}{B^{s}-1}\left(B^{s J_{L}}-B^{s\left(J_{0}-1\right)}\right) ; \\
& \sum_{j=J_{0}}^{J_{L}} B^{s j} \log B^{j}= \frac{B^{s}}{B^{s}-1} \log B\left(\left(J_{L}-\frac{1}{B^{s}-1}\right) B^{s J_{L}}\right. \\
&\left.-\left(\left(J_{0}-1\right)-\frac{1}{B^{s}-1}\right) B^{s\left(J_{0}-1\right)}\right) \\
& \sum_{j=J_{0}}^{J_{L}} B^{s j}\left(\log B^{j}\right)^{2} \\
&=\frac{B^{s}}{B^{s}-1}(\log B)^{2}\left(\left(\left(J_{L}-\frac{1}{B^{s}-1}\right)^{2}+\frac{B^{s}}{\left(B^{s}-1\right)^{2}}\right) B^{s J_{L}}\right. \\
&\left.-\left(\left(\left(J_{0}-1\right)-\frac{1}{B^{s}-1}\right)^{2}+\frac{B^{s}}{\left(B^{s}-1\right)^{2}}\right) B^{s\left(J_{0}-1\right)}\right)
\end{aligned}
$$

Corollary 2. Let

$$
V_{J_{0} ; J_{L}}(s)=\left(\sum_{j=J_{0}}^{J_{L}} B^{s j}\right)\left(\sum_{j=J_{0}}^{J_{L}} B^{s j}\left(\log B^{j}\right)^{2}\right)-\left(\sum_{j=J_{0}}^{J_{L}} B^{s j} \log B^{j}\right)^{2} .
$$


The we have

$V_{J_{0} ; J_{L}}(s)=\left(\frac{B^{s} \log B}{B^{s}-1}\right)^{2}\left[\frac{B^{s}}{\left(B^{s}-1\right)^{2}}\left(B^{s J_{L}}-B^{s\left(J_{0}-1\right)}\right)^{2}-B^{s\left(J_{L}+J_{0}-1\right)}\left(J_{L}-J_{0}+1\right)^{2}\right]$.

Moreover if $J_{0}=-J_{L}$

$V_{J_{L}}(s)=\left(\frac{B^{s} \log B}{B^{s}-1}\right)^{2}\left[\frac{B^{s}}{B^{s}-1}\left(B^{s J_{L}}-B^{s\left(-J_{L}-1\right)}\right)^{2}-\frac{1}{B^{s}} B^{s\left(2 J_{L}-1\right)}\left(2 J_{L}+1\right)^{2}\right]$,

so that

$$
\lim _{J_{L} \rightarrow \infty} B^{-2 s J_{L}} V_{J_{L}}(s)=\log ^{2} B \frac{B^{3 s}}{\left(B^{s}-1\right)^{4}} .
$$

\section{Appendix B. Auxiliary Results: Covariance terms}

Lemma B.1. Let $\tau_{B}(\Delta j)$ be defined as in (2.8). Hence we have for $4 p-\alpha_{0}>0$, $J_{0}<0$,

$$
\begin{gathered}
\Sigma_{0}\left(J_{L}\right):=\sum_{j=J_{0}}^{J_{L}} B^{2 j} \sum_{\Delta j=-J_{0}-j}^{J_{L}-j} \tau_{B}(\Delta j) B^{a_{0} \Delta j}=\frac{B^{2}}{B^{2}-1}\left(1+\widetilde{\tau}_{0}\right) B^{2 J_{L}}+o\left(B^{2 J_{L}}\right), \\
\Sigma_{1}\left(J_{L}\right):=\sum_{j=J_{0}}^{J_{L}} B^{2 j} \log B^{j} \sum_{\Delta j=-J_{0}-j}^{J_{L}-j} \tau_{B}(\Delta j) B^{a_{0} \Delta j} \\
\left(\text { B.2) } \quad=\frac{B^{2} \log B}{B^{2}-1}\left(\left(1+\widetilde{\tau}_{0}\right) J_{L}-\left(\frac{1}{B^{2}-1}\left(1+\widetilde{\tau}_{1}\right)\right)\right) B^{2 J_{L}}+o\left(B^{2 J_{L}}\right)\right. \\
\Sigma_{2}\left(J_{L}\right):=\sum_{j=J_{0}}^{J_{L}} B^{2 j} \log ^{2} B^{j} \sum_{\Delta j=-J_{0}-j}^{J_{L}-j} \tau_{B}(\Delta j) B^{a_{0} \Delta j}
\end{gathered}
$$

$$
=\frac{B^{2} \log ^{2} B}{B^{2}-1}\left(\left(1+\widetilde{\tau}_{0}\right) J_{L}^{2}-\left(\frac{2}{B^{2}-1}\left(1+\widetilde{\tau}_{1}\right)\right) J_{L}+\frac{B^{2}+1}{\left(B^{2}-1\right)^{2}}\left(1+\widetilde{\tau}_{2}\right)\right) B^{2 J_{L}}+o\left(B^{2 J_{L}}\right),
$$

where

$$
\begin{gathered}
\widetilde{\tau}_{0}:=\frac{2^{\left(4 p+1-\alpha_{0}\right)}}{\left(B^{\left(4 p+2-\alpha_{0}\right)}-1\right)} ; \\
\widetilde{\tau}_{1}:=2^{4 p+1-\alpha_{0}} \frac{\left(B^{4 p+4-\alpha_{0}}-1\right)}{\left(B^{4 p+2-\alpha_{0}}-1\right)^{2}} ; \\
\widetilde{\tau}_{2}:=2^{4 p+1-\alpha_{0}}\left(\frac{W_{p}(B)}{\left(B^{4 p-\alpha_{0}+1}-1\right)^{3}}\right),
\end{gathered}
$$

and

$W_{p}(B):=\frac{\left(B^{6} B^{4 p-\alpha_{0}}\left(B^{(P-1)}+1\right)+B^{4} B^{\left(4 p-\alpha_{0}\right)}\left(B^{3} B^{4 p-\alpha_{0}+1}-6\right)+B^{2}\left(B^{\left(4 p-\alpha_{0}\right)}+1\right)+1\right)}{B^{2}+1}$.

Moreover if we define

$$
Z_{J_{L}}:=\Sigma_{0}\left(J_{L}\right) \Sigma_{2}\left(J_{L}\right)-\Sigma_{1}^{2}\left(J_{L}\right)
$$


we have

$$
\lim _{J_{L}=0} B^{-4 J_{L}} Z_{J_{L}}:=\frac{B^{6} \log ^{2} B}{\left(B^{2}-1\right)^{4}}(1+\widetilde{\tau})
$$

where

$$
\widetilde{\tau}:=\frac{1}{B^{2}}\left(\left(B^{2}+1\right)\left(\widetilde{\tau}_{0}+\widetilde{\tau}_{2}+\widetilde{\tau}_{0} \widetilde{\tau}_{2}\right)+2 \widetilde{\tau}_{1}-\widetilde{\tau}_{1}^{2}\right)
$$

Proof. Let us call $P=\left(4 p+1-\alpha_{0}\right)$ and observe that:

$$
\begin{gathered}
\sum_{\Delta j=J_{0}-j}^{J_{L}-j} B^{\alpha_{0} \Delta j} \tau_{B}(\Delta j)-1 \\
=\sum_{\Delta j=J_{0}-j}^{J_{L}-j} B^{\Delta j}[\cosh (\Delta j \log B)]^{-P}-1=2^{P} \sum_{\Delta j=J_{0}-j}^{J_{L}-j} \frac{B^{\Delta j}}{\left(B^{\Delta j}+B^{-\Delta j}\right)^{P}}-1 \\
=2^{P}\left(\sum_{\Delta j=J_{0}-j}^{-1} \frac{1}{\left(B^{\Delta j\left(\frac{P-1}{P}\right)}+B^{\left.-\Delta j\left(\frac{P+1}{P}\right)\right)^{P}}\right.}+\sum_{\Delta j=1}^{J_{L}-j} \frac{1}{\left(B^{\Delta j\left(\frac{P-1}{P}\right)}+B^{-\Delta j\left(\frac{P+1}{P}\right)}\right)^{P}}\right),
\end{gathered}
$$

where we have considered the case $J_{0}<0$. Hence we have, from Proposition 2

$$
\begin{aligned}
\sum_{\Delta j=J_{0}-j}^{-1} \frac{1}{\left(B^{\Delta j\left(\frac{P-1}{P}\right)}+B^{-\Delta j\left(\frac{P+1}{P}\right)}\right)^{P}} & \simeq \sum_{\Delta j=1}^{j-J_{0}} B^{-(P+1) \Delta j} \\
& =\frac{1}{B^{(P+1)}-1}\left(1-B^{-(P+1)\left(j-J_{0}\right)}\right)
\end{aligned}
$$

while we have

$$
\begin{aligned}
\sum_{\Delta j=1}^{J_{L}-j} \frac{1}{\left(B^{\Delta j\left(\frac{P-1}{P}\right)}+B^{-\Delta j\left(\frac{P+1}{P}\right)}\right)^{P}} & \simeq \sum_{\Delta j=1}^{J_{L}-j} B^{-(P-1) \Delta j} \\
& =\frac{1}{B^{(P-1)}-1}\left(1-B^{-(P-1)\left(J_{L}-j\right)}\right),
\end{aligned}
$$

Consider now

$$
\begin{gathered}
\sum_{j=J_{0}}^{J_{L}} B^{2 j} \sum_{\Delta j=-J_{0}-j}^{J_{L}-j} B^{\Delta j} \tau_{B}(\Delta j) \\
=\sum_{j=J_{0}}^{J_{L}} B^{2 j}+\sum_{j=J_{0}} B^{2 j} \sum_{\Delta j=J_{0}-j}^{-1} 2^{P} B^{\Delta j(P+1)}+\sum_{j=1}^{J_{L}} B^{2 j} \sum_{\Delta j=1}^{J_{L}-j} 2^{P} B^{-\Delta j(P-1)} .
\end{gathered}
$$

We have, given that $P+1>0$, if $J_{0}<0$

$$
\sum_{j=J_{0}}^{-1} B^{2 j} \sum_{\Delta j=J_{0}-j}^{-1} B^{\Delta j(P+1)}
$$




$$
\begin{aligned}
& =\sum_{j=J_{0}}^{-1} B^{2 j} \frac{1}{B^{(P+1)}-1}\left(1-B^{-(P+1)\left(j-J_{0}\right)}\right) \\
& =\frac{1}{B^{(P+1)}-1}\left(\frac{B^{2}}{B^{2}-1}\left(B^{-2}-B^{2\left(J_{0}-1\right)}\right)-\frac{B^{(P+1) J_{0}}}{B^{P-1}-1}\left(B^{(1-P)\left(J_{0}-1\right)}-B^{-(1-P)}\right)\right) \\
& =o\left(B^{2 J_{L}}\right) .
\end{aligned}
$$

On the other hand,

$$
\begin{gathered}
\sum_{j=1}^{J_{L}} B^{2 j} \sum_{\Delta j=1}^{J_{L}-j} B^{-\Delta j(P-1)} \\
=\sum_{j=J_{0}}^{J_{L}} B^{2 j} \frac{1}{B^{(P-1)}-1}\left(1-B^{-(P-1)\left(J_{L}-j\right)}\right) \\
=\frac{1}{B^{(P-1)}-1}\left(\frac{B^{2}}{B^{2}-1} B^{2 J_{L}}-\frac{B^{(P+1)}}{B^{(P+1)}-1} B^{2 J_{L}}\right)+o\left(B^{2 J_{L}}\right) \\
=\left(\frac{B^{2}}{\left(B^{2}-1\right)\left(B^{P+1}-1\right)}\right) B^{2 J_{L}}+o\left(B^{2 J_{L}}\right) .
\end{gathered}
$$

Hence we have

$$
\begin{aligned}
& \sum_{j=J_{0}}^{J_{L}} B^{2 j} \sum_{\Delta j=-J_{0}-j}^{J_{L}-j} B^{\Delta j} \tau_{B}(\Delta j) \\
= & \frac{B^{2}}{B^{2}-1} B^{2 J_{L}}\left(1+\frac{2^{P}}{\left(B^{P+1}-1\right)}\right)+o\left(B^{J_{L}}\right) \\
= & \frac{B^{2}}{B^{2}-1} B^{2 J_{L}}\left(1+\widetilde{\tau}_{0}\right)+o\left(B^{J_{L}}\right),
\end{aligned}
$$

Similar calculations lead to

$$
\begin{aligned}
& \qquad \sum_{j=J_{0}}^{J_{L}} B^{2 j} \log B^{j} \sum_{\Delta j=-J_{0}-j}^{J_{L}-j} B^{\Delta j} \tau_{B}(\Delta j) \\
& =\sum_{j=J_{0}}^{J_{L}} B^{2 j} \log B^{j}+2^{P}\left(\sum_{j=J_{0}}^{-1} B^{2 j} \log B^{j} \sum_{\Delta j=J_{0}-j}^{-1} B^{\Delta j(P+1)}+\sum_{j=1}^{J_{L}} B^{2 j} \log B^{j} \sum_{\Delta j=1}^{J_{L}-j} B^{-\Delta j(P-1)}\right) \\
& \text { where } \\
& =\sum_{j=1}^{J_{L}} B^{2 j} \log B^{j}\left(\frac{1}{B^{(P-1)}-1}\left(1-B^{-\left(J_{L}-j\right)(P-1)}\right)\right) \\
& =\frac{\log B}{B^{(P-1)}-1} B^{2 J_{L}}\left(\frac{B^{2}\left(B^{P-1}-1\right)}{\left(B^{2}-1\right)\left(B^{P+1}-1\right)} J_{L}^{j}-\frac{B^{2}\left(B^{2+(P+1)}-1\right)\left(B^{(P-1)}-1\right)}{\left(B^{2}-1\right)^{2}\left(B^{(P+1)}-1\right)^{2}}\right)+o\left(B^{2 J_{L}}\right) \\
& =\frac{B^{2} \log B}{B^{2}-1} B^{2 J_{L}}\left(\frac{1}{\left(B^{P+1}-1\right)} J_{L}-\frac{\left(B^{2+(P+1)}-1\right)}{\left(B^{2}-1\right)\left(B^{(P+1)}-1\right)^{2}}\right)+o\left(B^{2 J_{L}}\right)
\end{aligned}
$$


while, if $J_{0}<0$

$$
\sum_{j=J_{0}}^{-1} B^{2 j} \log B^{j} \sum_{\Delta j=J_{0}-j}^{-1} B^{\Delta j(P+1)}=o\left(B^{2 J_{L}}\right) .
$$

We hence obtain

$$
\begin{gathered}
\sum_{j=J_{0}}^{J_{L}} B^{2 j} \log B^{j} \sum_{\Delta j=-J_{0}-j}^{J_{L}-j} B^{\Delta j} \tau_{B}(\Delta j) \\
=\frac{B^{2} \log B B^{2 J_{L}}}{B^{2}-1}\left(J_{L}\left(1+\widetilde{\tau}_{0}\right)-\frac{1}{B^{2}-1}\left(1+\frac{2^{P}\left(B^{2+(P+1)}-1\right)}{\left(B^{(P+1)}-1\right)^{2}}\right)\right)+o\left(B^{2 J_{L}}\right) \\
=\frac{B^{2} \log B B^{2 J_{L}}}{B^{2}-1}\left(J_{L}\left(1+\widetilde{\tau}_{0}\right)-\frac{1}{B^{2}-1}\left(1+\widetilde{\tau}_{1}\right)\right)+o\left(B^{2 J_{L}}\right) .
\end{gathered}
$$

Furthermore, we have:

$$
\begin{gathered}
\sum_{j=J_{0}}^{J_{L}} B^{2 j} \log ^{2} B^{j} \sum_{\Delta j=-J_{0}-j}^{J_{L}-j} B^{\Delta j} \tau_{B}(\Delta j) \\
=\sum_{j=J_{0}}^{J_{L}} B^{2 j} \log ^{2} B^{j}+2^{P}\left(\sum_{j=J_{0}}^{-1} B^{2 j} \log ^{2} B^{j} \sum_{\Delta j=J_{0}-j}^{-1} B^{\Delta j(P+1)}+\sum_{j=1}^{J_{L}} B^{2 j} \log ^{2} B^{j} \sum_{\Delta j=1}^{J_{L}-j} B^{-\Delta j(P-1)}\right) .
\end{gathered}
$$

We observe that

$$
\begin{aligned}
\sum_{j=1}^{J_{L}} B^{2 j} \log ^{2} B^{j} \sum_{\Delta j=1}^{J_{L}-j} B^{-\Delta j(P-1)} & \frac{1}{B^{P-1}-1}\left(\frac{B^{2} \log ^{2} B}{B^{2}-1} B^{2 J_{L}}\left(\left(J_{L}-\frac{1}{B^{2}-1}\right)^{2}+\frac{B^{2}}{\left(B^{2}-1\right)^{2}}\right)-B^{-(P-1) J_{L}} \sum_{j=1}^{J_{L}} B^{(P+1) j} \log ^{2} B^{j}\right) \\
= & \frac{B^{2} \log ^{2} B}{\left(B^{2}-1\right)} B^{2 J_{L}}\left(J_{L}^{2}\left(\frac{1}{\left(B^{(P+1)}-1\right)}\right)-2 \frac{1}{\left(B^{2}-1\right)} J_{L}\left(\frac{\left(B^{2+(P+1)}-1\right)}{\left(B^{(P+1)}-1\right)^{2}}\right)\right. \\
& \left.+\left(\frac{B^{2}+1}{\left(B^{2}-1\right)^{2}} \frac{W_{p}(B)}{\left(B^{P+1}-1\right)^{3}}\right)\right)+o\left(B^{2 J_{L}}\right) \\
= & \frac{B^{2} \log ^{2} B}{\left(B^{2}-1\right)} B^{2 J_{L}}\left(\widetilde{\tau}_{0} J_{L}^{2}-2 \frac{\widetilde{\tau}_{1}}{\left(B^{2}-1\right)} J_{L}+\frac{B^{2}+1}{\left(B^{2}-1\right)^{2}} \widetilde{\tau}_{2}\right)+o\left(B^{2 J_{L}}\right)
\end{aligned}
$$

where

$W_{p}(B)=\frac{\left(B^{6} B^{P-1}\left(B^{(P-1)}+1\right)+B^{4} B^{(P-1)}\left(B^{3} B^{P}-6\right)+B^{2}\left(B^{(P-1)}+1\right)+1\right)}{B^{2}+1}$.

On the other hand we have

$$
\sum_{j=J_{0}}^{-1} B^{2 j} \log ^{2} B^{j} \sum_{\Delta j=J_{0}-j}^{-1} B^{\Delta j(P+1)}=o\left(B^{2 J_{L}}\right),
$$

so that

$$
\sum_{j=J_{0}}^{J_{L}} B^{2 j} \log ^{2} B^{j} \sum_{\Delta j=-J_{0}-j}^{J_{L}-j} B^{\Delta j} \tau_{B}(\Delta j)
$$




$$
=\frac{B^{2} \log ^{2} B}{\left(B^{2}-1\right)} B^{2 J_{L}}\left(\left(1+\widetilde{\tau}_{0}\right) J_{L}^{2}-\frac{2\left(1+\widetilde{\tau}_{1}\right)}{\left(B^{2}-1\right)} J_{L}+\frac{B^{2}+1}{\left(B^{2}-1\right)^{2}}\left(1+\widetilde{\tau}_{2}\right)\right)+o\left(B^{2 J_{L}}\right) .
$$

Hence we have, from Corollary 2, that

$$
\begin{aligned}
Z_{J_{L}}= & \frac{B^{4} \log ^{2} B}{\left(B^{2}-1\right)^{2}} B^{4 J_{L}}\left(\left(\left(1+\widetilde{\tau}_{0}\right) J_{L}^{2}-\frac{2\left(1+\widetilde{\tau}_{1}\right)}{\left(B^{2}-1\right)} J_{L}+\frac{B^{2}+1}{\left(B^{2}-1\right)^{2}}\left(1+\widetilde{\tau}_{2}\right)\right)\left(1+\widetilde{\tau}_{0}\right)\right. \\
& \left.-\left(J_{L}\left(1+\widetilde{\tau}_{0}\right)-\frac{1}{B^{2}-1}\left(1+\widetilde{\tau}_{1}\right)\right)^{2}\right) \\
= & \frac{B^{6} \log ^{2} B}{\left(B^{2}-1\right)^{4}} B^{4 J_{L}}\left(1+\left(\frac{\left(B^{2}+1\right)}{B^{2}}\left(\widetilde{\tau}_{0}+\widetilde{\tau}_{2}+\widetilde{\tau}_{0} \widetilde{\tau}_{2}\right)+\frac{2 \widetilde{\tau}_{1}-\widetilde{\tau}_{1}^{2}}{B^{2}}\right)\right) \\
= & \frac{B^{6} \log ^{2} B}{\left(B^{2}-1\right)^{4}} B^{4 J_{L}}(1+\widetilde{\tau}),
\end{aligned}
$$

as claimed.

\section{Appendix C. Auxiliary Results: Cumulants}

Lemma C.1. Let $A_{j}$ and $B_{j}$ be defined as in 4.10) and 4.11). As $J_{L} \rightarrow \infty$

$$
\begin{aligned}
\frac{1}{B^{4 J_{L}}} \operatorname{cum}\left\{\sum_{l_{1}}\left(A_{j_{1}}+B_{j_{1}}\right)\right. & \left., \sum_{l_{2}}\left(A_{j_{2}}+B_{j_{2}}\right), \sum_{l_{3}}\left(A_{j_{3}}+B_{j_{3}}\right), \sum_{l_{4}}\left(A_{j_{4}}+B_{j_{4}}\right)\right\} \\
= & O_{J_{L}}\left(\frac{J_{L}^{4} \log ^{4} B}{B^{2 J_{L}}}\right) .
\end{aligned}
$$

Proof. It is readily checked (see also [12]) that

$$
\operatorname{cum}\left\{\widehat{C}_{l}, \widehat{C}_{l}, \widehat{C}_{l}, \widehat{C}_{l}\right\}=O\left(l^{-3} l^{-4 \alpha_{0}}\right) .
$$

Let us compute:

$$
\begin{gathered}
C_{j_{1}, j_{2}, j_{3} j_{4}}^{4}=\operatorname{cum}\left(\frac{\sum_{k} \beta_{j_{1} k ; p}^{2}}{N_{j_{1}} G_{0} K_{j_{1}}^{M}\left(\alpha_{0}\right)}, \frac{\sum_{k} \beta_{j_{2} k ; p}^{2}}{N_{j_{2}} G_{0} K_{j_{2}}^{M}\left(\alpha_{0}\right)}, \frac{\sum_{k} \beta_{j_{3} k ; p}^{2}}{N_{j_{3}} G_{0} K_{j_{3}}^{M}\left(\alpha_{0}\right)}, \frac{\sum_{k} \beta_{j_{4} k ; p}^{2}}{N_{j_{4}} G_{0} K_{j_{4}}^{M}\left(\alpha_{0}\right)}\right) \\
=\left(\prod_{i=1}^{4} \frac{1}{N_{j_{i}} G_{0} K_{j_{i}}^{M}\left(\alpha_{0}\right)}\right) \operatorname{cum}\left(\sum_{k} \beta_{j_{1} k ; p}^{2}, \sum_{k} \beta_{j_{2} k ; p}^{2}, \sum_{k} \beta_{j_{3} k ; p}^{2}, \sum_{k} \beta_{j_{4} k ; p}^{2}\right) \\
=\sum_{l_{1}, l_{2}, l_{3}, l_{4}}\left(\prod_{i=1}^{4} \frac{f_{p}^{2}\left(\frac{l_{i}}{B^{j_{i}}}\right)\left(2 l_{i}+1\right)}{N_{j_{i}} G_{0} K_{j_{i}}\left(\alpha_{0}\right)}\right) \operatorname{cum}\left(\widehat{C}_{l_{1}}, \widehat{C}_{l_{2}}, \widehat{C}_{l_{3}}, \widehat{C}_{l_{4}}\right) \\
=\sum_{l}(2 l+1)^{4}\left(\prod_{i=1}^{4} \frac{f_{p}^{2}\left(\frac{l_{i}}{B^{j_{i}}}\right)}{N_{j_{i}} G_{0} K_{j_{i}}\left(\alpha_{0}\right)}\right) \operatorname{cum}\left(\widehat{C}_{l}, \widehat{C}_{l}, \widehat{C}_{l}, \widehat{C}_{l}\right)+o\left(B^{-4 j}\right) \\
=O\left(\sum_{l}\left(\prod_{i=1}^{4} B^{\left(\alpha_{0}-2\right) j_{i}} f_{p}^{2}\left(\frac{l_{i}}{B^{j_{i}}}\right)\right) B^{\left(2-4 \alpha_{0}\right) j}\left(l^{1-4 \alpha_{0}}\right)\right) \\
=O\left(B^{-6 j} \prod_{i=1}^{4} \delta_{j}^{j_{i}}\right) .
\end{gathered}
$$


Then we have

$$
\begin{aligned}
& \operatorname{cum}\left\{\frac{\widehat{G}_{J_{0}, J_{L}}^{M}\left(\alpha_{0}\right)}{G_{0}}, \frac{\widehat{G}_{J_{0}, J_{L}}^{M}\left(\alpha_{0}\right)}{G_{0}}, \frac{\widehat{G}_{J_{0}, J_{L}}^{M}\left(\alpha_{0}\right)}{G_{0}}, \frac{\widehat{G}_{J_{0}, J_{L}}^{M}\left(\alpha_{0}\right)}{G_{0}}\right\} \\
= & O\left(\frac{1}{B^{8 J_{L}}} \sum_{j_{1} j_{2} j_{3} j_{4}} N_{j_{1}} N_{j_{2}} N_{j_{3}} N_{j_{4}} C_{j_{1}, j_{2}, j_{3} j_{4}}^{4}\right) \\
= & O\left(\frac{1}{B^{8 J_{L}}} \sum_{j} B^{2 j}\right)=O\left(B^{-6 J_{L}}\right) .
\end{aligned}
$$

As in 12 and 13, the proof can be divided into 5 cases, corresponding respectively to

$$
\begin{aligned}
& \frac{1}{B^{4 J_{L}}} \operatorname{cum}\left\{\sum_{j_{1}} A_{j_{1}}, \sum_{j_{2}} A_{j_{2}}, \sum_{j_{3}} A_{j_{3}}, \sum_{j_{4}} A_{j_{4}}\right\}, \frac{1}{B^{4 J_{L}}} \operatorname{cum}\left\{\sum_{j_{1}} B_{j_{1}}, \sum_{j_{2}} B_{j_{2}}, \sum_{j_{3}} B_{j_{3}}, \sum_{j_{4}} B_{j_{4}}\right\} \\
& \frac{1}{B^{4 J_{L}}} \operatorname{cum}\left\{\sum_{j_{1}} A_{j_{1}}, \sum_{j_{2}} B_{j_{2}}, \sum_{j_{3}} B_{j_{3}}, \sum_{j_{4}} B_{j_{4}}\right\}, \frac{1}{B^{4 J_{L}}} \operatorname{cum}\left\{\sum_{j_{1}} A_{j_{1}}, \sum_{j_{2}} A_{j_{2}}, \sum_{j_{3}} B_{j_{3}}, \sum_{j_{4}} B_{j_{4}}\right\}
\end{aligned}
$$

and

$$
\frac{1}{B^{4 J_{L}}} \operatorname{cum}\left\{\sum_{j_{1}} A_{j_{1}}, \sum_{j_{2}} A_{j_{2}}, \sum_{j_{3}} A_{j_{3}}, \sum_{j_{4}} B_{j_{4}}\right\},
$$

where we have used 4.10, 4.11. We have for instance

$$
\begin{aligned}
& \frac{1}{B^{4 J_{L}}} \operatorname{cum}\left\{\sum_{j_{1}} A_{j_{1}}, \sum_{j_{2}} A_{j_{2}}, \sum_{j_{3}} A_{j_{3}}, \sum_{j_{4}} A_{j_{4}}\right\} \\
= & O\left(\frac{1}{B^{4 J_{L}}} \sum_{j_{1}, j_{2} j_{3}, j_{4}} \prod_{i=1}^{4}\left(B^{2 j_{i}} \log B^{j_{i}}\right) C_{j_{1}, j_{2}, j_{3} j_{4}}^{4}\right) \\
= & O\left(\frac{1}{B^{4 J_{L}}} \sum_{j} B^{8 j} \log ^{4} B^{j} B^{-6 j}\right)=O\left(\frac{1}{B^{4 J_{L}}} \sum_{j} \log ^{4} B^{j} B^{2 j}\right)=O\left(\frac{\log ^{4} B^{J_{L}}}{B^{2 J_{L}}}\right) ;
\end{aligned}
$$

and

$$
\begin{aligned}
& \frac{1}{B^{4 J_{L}}} \operatorname{cum}\left\{\sum_{j_{1}} B_{j_{1}}, \sum_{j_{2}} B_{j_{2}}, \sum_{j_{3}} B_{j_{3}}, \sum_{j_{4}} B_{j_{4}}\right\} \\
= & \frac{1}{B^{4 J_{L}}}\left\{\sum_{j} B^{2 j} \log B^{j}\right\} \operatorname{cum}\left\{\frac{\widehat{G}_{J_{L}}\left(\alpha_{0}\right)}{G_{0}}, \frac{\widehat{G}_{J_{L}}\left(\alpha_{0}\right)}{G_{0}}, \frac{\widehat{G}_{J_{L}}\left(\alpha_{0}\right)}{G_{0}}, \frac{\widehat{G}_{J_{L}}\left(\alpha_{0}\right)}{G_{0}}\right\} \\
= & O\left(\log ^{4} B^{J_{L}} B^{-2 J_{L}}\right) ;
\end{aligned}
$$

The proof for the remaining terms is entirely analogous, and hence omitted. 


\section{References}

[1] Adler, R. J., Taylor, J. E. (2007) Random Fields and Geometry, Springer.

[2] Anderes, E., (2010) On the Consistent Separation of Scale and Variance for Gaussian Random Fields, Annals of Statistics, 38, no. 2, 870-893

[3] Baldi, P. and Marinucci, D. (2007) Some Characterizations of the Spherical Harmonics Coefficients for Isotropic Random Fields, Statistics and Probability Letters, 77, 490-496

[4] Baldi, P., Kerkyacharian, G., Marinucci, D. and Picard, D. (2009) Asymptotics for Spherical Needlets, Annals of Statistics, Vol. 37, No. 3, 1150-1171

[5] Baldi, P., Kerkyacharian, G., Marinucci, D. and Picard, D. (2009) Subsampling Needlet Coefficients on the Sphere, Bernoulli, Vol. 15, 438-463, arXiv: 0706.4169

[6] Baldi, P., Kerkyacharian, G., Marinucci, D. and Picard, D. (2009) Adaptive Density Estimation for directional Data Using Needlets, Annals of Statistics, Vol. 37, No. 6A, 33623395, arXiv: 0807.5059

[7] Brillinger, D.R. (1975) Statistical inference for stationary point processes. Stochastic processes and related topics (Proc. Summer Res. Inst. Statist. Inference for Stochastic Processes, Indiana Univ., Bloomington, Ind.), Vol.1, 55-99.

[8] Cabella, P. and Marinucci, D. (2009) Statistical Challenges in the Analysis of Cosmic Microwave Background Radiation, Annals of Applied Statistics, Vol.2, pp.61-95

[9] Dodelson, S. (2003) Modern Cosmology, Academic Press

[10] Doroshkevich, A.G., Verkhodanov, O.V., Naselsky, P.D., Kim, J, Novikov, D.I., Turchaninov V. I., Novikov, I.D., Chiang, L., Hansen, M. (2011), The GaussLegendre Sky Pixelization for the CMB polarization (GLESP-pol). Errors due to pixelization of the CMB sky, Int. J. Mod. Phys. D20:1053-1078

[11] Durastanti, C., Geller, D., Marinucci, D., (2011), Adaptive Nonparametric Regression of Spin Fiber Bundles on the Sphere, Journal of Multivariate Analysis, Vol. 104, Issue 1, pp. 16-38

[12] Durastanti, C., Lan, X., Marinucci, D., (2011), Gaussian Semiparametric Estimates on the Unit Sphere, Bernoulli, in press

[13] Durastanti, C., Lan, X., Marinucci, D., (2011), Needlet-Whittle Estimates on the Unit Sphere, Electronic Journal of Statistics, in press

[14] Durastanti, C. (2011) Semiparametric and Nonparametric Estimation on the Sphere by Needlet Methods, $\mathrm{PhD}$ thesis, University of Pavia.

[15] Faÿ, G., F. Guilloux, M. Betoule, J.-F. Cardoso, J. Delabrouille, M. Le Jeune (2008), CMB Power Spectrum Estimation Using Wavelets, Physical Review D, D78:083013

[16] Geller, D., Hansen, F.K., Marinucci, D., Kerkyacharian, G. and Picard, D. (2008), Spin Needlets for Cosmic Microwave Background Polarization Data Analysis, Physical Review D, D78:123533, arXiv:0811.2881

[17] Geller, D. and Marinucci, D. (2010) Spin Wavelets on the Sphere, Journal of Fourier Analysis and its Applications, n.6, 840-884, arXiv: 0811.2835

[18] Geller, D. and Marinucci, D. (2011) Mixed Needlets, Journal of Mathematical Analysis and Applications, n.375, 610-630.

[19] Geller, D., Lan, X. and Marinucci, D. (2009) Spin Needlets Spectral Estimation, Electronic Journal of Statistics, Vol. 3, 1497-1530

[20] Geller, D. and Mayeli, A. (2009) Continuous Wavelets on Manifolds, Math. Z., Vol. 262, pp. 895-927, arXiv: math/0602201

[21] Geller, D. and Mayeli, A. (2009) Nearly Tight Frames and Space-Frequency Analysis on Compact Manifolds, Math. Z., Vol, 263 (2009), pp. 235-264, arXiv: 0706.3642

[22] Geller, D. and Mayeli, A. (2009) Besov Spaces and Frames on Compact Manifolds, Indiana Univ. Math. J., Vol. 58, pp. 2003-2042, arXiv:0709.2452

[23] Gorski, K.M., Hivon, E, Banday, A.J., Wandelt, B.D., Hansen, F.K., Reinecke, M, Bartelman, M. (2005), HEALPix - a Framework for High Resolution Discretization, and Fast Analysis of Data Distributed on the Sphere, Astrophys. J. 622:759-771

[24] Hamann, J. and Wong, Y. Y. Y. (2008) The Effects of Cosmic Microwave Background (CMB) Temperature Uncertainties on Cosmological Parameter Estimation, Journal of Cosmology and Astroparticle Physics, Issue 03, 025

[25] Kerkyacharian, G., Pham Ngoc, T.M., Picard, D. (2011), Localized Spherical Deconvolution, Annals of Statistics, Vol. 39, no. 2, 1042-1068 
[26] Kim, P.T., Koo, J.-Y. (2002) Optimal Spherical Deconvolution, Journal of Multivariate Analysis, 80, 21-42

[27] Kim, P.T., Koo, J.-Y., Luo, Z.-M. (2009) Weyl Eigenvalue Asymptotics and Sharp Adaptation on Vector Bundles, Journal of Multivariate Analysis, 100, 1962-1978

[28] Koo, J.-Y. and Kim, P.T. (2008) Sharp Adaptation for Spherical Inverse Problems with Applications to Medical Imaging, Journal of Multivariate Analysis, 99, 165-190

[29] Lan, X. and Marinucci, D. (2008) The Needlets Bispectrum, Electronic Journal of Statistics, Vol. 2, pp.332-367

[30] Lan, X. and Marinucci, D. (2009) On the Dependence Structure of Wavelet Coefficients for Spherical Random Fields, Stochastic Processes and their Applications, 119, 3749-3766, arXiv:0805.4154

[31] Larson, D. et al. (2011) Seven-Year Wilkinson Microwave Anisotropy Probe (WMAP) Observations: Power Spectra and WMAP-Derived Parameters, Astrophysical Journal Supplement Series, 192:16.

[32] Leonenko, N. (1999) Limit Theorems for Random Fields with Singular Spectrum, Mathematics and its Applications, 465. Kluwer Academic Publishers, Dordrecht

[33] Leonenko, N. and Sakhno L. (2012) On Spectral Representations of Tensor Random Fields on the Sphere, Stoch. Anal. Appl. 30, no. 1, 44-66, arXiv:0912.3389

[34] Loh, W.-L. (2005) Fixed-Domain Asymptotics for a Subclass of Matérn-type Gaussian Random Fields, Annals of Statistics, Vol. 33, No. 5, 2344-2394

[35] Malyarenko, A. (2011), Invariant Random Fields in Vector Bundles and Applications to Cosmology, Ann. Inst. Henri Poincaré Probab. Stat. 47 (2011), no. 4, 1068-1095, arXiv: 0907.4620

[36] Marinucci, D. and Peccati, G. (2010), Ergodicity and Gaussianity for Spherical Random Fields. Journal of Mathematical Physics 51, 043301

[37] Marinucci, D. and Peccati, G. (2011) Random Fields on the Sphere. Representation, Limit Theorem and Cosmological Applications, Cambridge University Press.

[38] Marinucci, D., Pietrobon, D., Balbi, A., Baldi, P., Cabella, P., Kerkyacharian, G., Natoli, P. Picard, D., Vittorio, N., (2008) Spherical Needlets for CMB Data Analysis, Monthly Notices of the Royal Astronomical Society, Volume 383, Issue 2, pp. 539-545

[39] Mayeli, A. (2010), Asymptotic Uncorrelation for Mexican Needlets, J. Math. Anal. Appl. Vol. 363, Issue 1, pp. 336-344, arXiv: 0806.3009

[40] Narcowich, F.J., Petrushev, P. and Ward, J.D. (2006a) Localized Tight Frames on Spheres, SIAM Journal of Mathematical Analysis Vol. 38, pp. 574-594

[41] Narcowich, F.J., Petrushev, P. and Ward, J.D. (2006b) Decomposition of Besov and Triebel-Lizorkin Spaces on the Sphere, Journal of Functional Analysis, Vol. 238, 2, 530-564

[42] Nourdin, I. and Peccati, G. (2009) Stein's Method on Wiener Chaos, Probability Theory and Related Fields, 145, no. 1-2, 75-118.

[43] Pietrobon, D., Balbi, A., Marinucci, D. (2006) Integrated Sachs-Wolfe Effect from the Cross Correlation of WMAP3 Year and the NRAO VLA Sky Survey Data: New Results and Constraints on Dark Energy, Physical Review D, id. D:74, 043524

[44] Pietrobon, D., Amblard, A., Balbi, A., Cabella, P., Cooray, A., Marinucci, D. (2008) Needlet Detection of Features in WMAP CMB Sky and the Impact on Anisotropies and Hemispherical Asymmetries, Physical Review D, D78 103504

[45] Pietrobon, D., Cabella, P., Balbi, A., de Gasperis, G., Vittorio, N. (2009) Constraints on Primordial non-Gaussianity from a Needlet Analysis of the WMAP-5 Data, arXiv: 0812.2478, Monthly Notices of the Royal Astronomical Society, Volume 396, Issue 3, pp. 16821688

[46] Robinson, P.M. (1995), Gaussian Semiparametric Estimation for Long Range Dependence, The Annals of Statistics, 22, 1630-1661.

[47] Rudjord, O., Hansen, F.K., Lan, X., Liguori, M. Marinucci, D., Matarrese, S. (2010) Directional Variations of the Non-Gaussianity Parameter $f_{N L}$, Astrophysical Journal, Volume 708, Issue 2, pp. 1321-1325, arXiv: 0906.3232

[48] Scodeller, S., Rudjord, O. Hansen, F.K., Marinucci, D., Geller, D. and Mayeli, A. (2011) Introducing Mexican needlets for CMB analysis: Issues for practical applications and comparison with standard needlets, Astrophysical Journal, 733, 121

[49] Stein, E.M. and Weiss, G. (1971) Introduction to Fourier Analysis on Euclidean Spaces. Princeton University Press 
[50] Varshalovich, D.A., A.N. Moskalev, and V.K. Khersonskii (1988), Quantum Theory of Angular Momentum, World Scientific

(C. Durastanti) University of Rome "Tor VergatA"

E-mail address, C. Durastanti: durastan@mat.uniroma2.it

(X. Lan) University of Science and Technology of China (Hefei, Anhui, China, 230026)

E-mail address, X. Lan: xhlan@ustc.edu.cn 